\title{
IR/Visible Polarization Measurements of Scattered Solar Radiation from Clouds ${ }^{1}$
}

\author{
A.J. LePage, A.T. Stair, Jr., R.J. Jordano, P.C. Joss \\ Visidyne, Inc. \\ 10 Corporate Place \\ South Bedford Street \\ Burlington, MA 01803 \\ (978) 273-2820 \\ lepage@visidyne.com, ats@visidyne.com,jordano@visidyne.com, joss@mitlns.mit.edu \\ J. Devore \\ Visidyne, Inc. \\ 5951 Encina Road, Suite 208 \\ Goleta, CA 93117 \\ (805) 683-4277 \\ devore@visidyne.com \\ J.A. Kristl \\ Stewart Radiance Laboratory \\ SDL/USU \\ 139 The Great Road \\ Bedford, MA 01730 \\ jkristl@sysdiv.sdl.usu.edu \\ B.P. Sanford, J.H. Schummers \\ AFRL/VSBC \\ 29 Randolph Road \\ Hanscom AFB, MA 01731 \\ sandford@plh.af.mil, schummers@plh.af.mil
}

\begin{abstract}
During 1997 and 1998 a series of flights were made using the FISTA aircraft which carried instruments to obtain polarization measurements of solar radiation scattered from clouds. Polarization measurements were simultaneously obtained at selected infrared and visible wavelength bands in an effort to differentiate between clouds of various compositions.
\end{abstract}

An analysis of the 1997 data indicates that the degree of polarization from an apparently homogeneous deck of clouds can vary markedly at a given scattering angle. This can be caused by differences in the source of scene illumination, the spatial variability in scattering properties of the clouds, as well as wavelength-dependent contributions from scattering in the clear atmosphere above the cloud deck. While our observations generally resemble our expectations based on single-scattering theoretical models, future efforts to incorporate the observations made during the 1997 and 1998 FISTA flights and the effects of multiple scattering into these models are planned.

\footnotetext{
${ }^{1}$ U.S. Government work not protected by U.S. copyright.
}

TABLE OF CONTENTS

1. INTRODUCTION

2. THEORY

3. INSTRUMENTATION

4. OBSERVATIONS

5. COMPARISON OF THEORY \& OBSERVATIONS

6. CONCLUSIONS

7. ACKNOWLEDGEMENTS

8. REFERENCES

9. BIOGRAPHY

\section{INTRODUCTION}

In 1997 and 1998 a series of flights were made using the FISTA (Flying Infrared Signatures Technology Aircraft) aircraft to obtain polarization measurements of solar radiation scattered from clouds in a number of infrared and visible wavelength bands simultaneously. The immediate purpose of this investigation is to obtain the data required to characterize the polarization properties of various atmospheric phenomena with applications towards background clutter mitigation, the ability to differentiate 
between clouds of various compositions (e.g. ice versus water clouds), and the verification of theoretical polarization models.

Computer-based polarization models that we have developed using single scattering theory indicate that differences in cloud composition, for example, can be best determined by making polarization measurements at wavelength bands that correspond to the shoulders of infrared absorption bands of water. While we originally intended to make such measurements at a variety of wavelengths, emphasis for the analysis of the 1997 data has been placed on the water absorption band centered at $3 \mu \mathrm{m}$ due to the ready availability of the appropriate instrumentation and filters.

Ultimately these measurements and models will be used to design instruments, choose appropriate wavelength bands, and plan experiments for future air- and space-based instrument platforms such as those envisioned for RAMOS (Russian American Observation Satellite).

For the initial round of FISTA flights carried out in July and September of 1997, existing instruments were modified with the addition of the appropriate polarization filters to make measurements in a range of wavelength bands from $\mathrm{mid} /$ long-wave infrared (M/LWIR) to the visible. A lidar system was also employed to provide data on the properties of the clouds observed such as their altitude, gross scattering properties, and composition.

Experience gained from the 1997 flights has allowed further modifications to be made to these instruments and the experiment design for a second series of flights carried out during September and October of 1998. These changes along with some new instrumentation were hoped to improve the quality of the new data collected and allow us to obtain a more synoptic view of the scattering environment observed.

In this paper we will review the theory of the polarization of light scattered from clouds and our initial efforts to produce a Monte Carlo computer model. We will then discuss our experiment plan (which was based on our model predictions), the capabilities and limitations of the instrumentation employed during the 1997 FISTA flights as well as a sample of the observations actually made. Finally we will compare our observations with the predictions made by our models and outline changes that were made in the experiment plan for the 1998 FISTA flights.

\section{THEORY}

The degree and angle of linear polarization of radiation scattered by clouds in the infrared absorption bands of water (e.g. at $3 \mu \mathrm{m}$ ) can be measured by use of aircraft, satellites, or ground-based instrumentation. Such measurements have at least two potentially important applications which are currently the subject of a patent application by Visidyne,
Inc. First, this technique affords a method of discriminating between clouds and other sources of radiation, and can thus provide a powerful technique for clutter mitigation. Second, the polarization signature contains substantial information regarding the phase (water, ice, or a mixture) of the particles at the tops of clouds.

Information regarding the phase of the cloud particles, combined with data on the altitude of the cloud tops, has, in turn, a number of potentially important scientific applications. In particular, determination of the glaciation level in clouds via remote sensing techniques would be of considerable importance to aviation interests, for use in numerical weather prediction, and for incorporation into global climate models.

There are certain types of clouds that are of particular interest for carrying out polarization measurements. These include:

(a) Clouds that are known to be composed either entirely of water droplets or ice crystals. The former include lowaltitude trade cumuli, which are common over the tropical oceans year round. The latter include well-developed baroclinic regions (which are most common at mid-latitudes during the winter months in each hemisphere), the anvils of mature thunderstorms (which commonly occur in many mid-latitude regions during the spring and summer seasons especially during mid- to late afternoon hours and in most tropical regions throughout the year), and the eyewalls of tropical cyclones (which are most commonly found in the vicinity of the intertropical convergence zone during the late summer and early autumn months in each hemisphere). Since the phase of the cloud particles is known, the measured polarization signals can be used to provide empirical confirmation of the signals predicted theoretically for each particle type.

(b) Mixed-phase clouds, particularly those that may contain regions of highly supercooled water droplets. The most easily identifiable clouds of this type will be clouds associated with thunderstorms in their formative stages. These include cumulus congestus and cumulonimbus with immature anvils which can be found where thunderstorms frequently occur. We note that such clouds have a great deal of vertical structure. Therefor if they are viewed from an oblique angle relative to the local nadir, considerable information concerning the phase of the cloud particles as a function of altitude may be gained, even though only scattering from the outer surface of the cloud can be measured.

\section{Cloud Polarization}

Let $P\left(\theta_{S}, \theta_{0}, \varphi_{0}\right)$ and $\Omega\left(\theta_{S}, \theta_{0}, \varphi_{0}\right)$ be the degree of linear polarization and the direction of the polarization vector (relative to some reference direction), respectively, for radiation incident from a source of unpolarized light (such as sunlight at polar angle $\theta_{\mathrm{S}}$ in the $\mathrm{x}-\mathrm{z}$ plane and scattered 
toward an observer in the direction $\theta_{0}, \varphi_{0}$ where the $+z$-axis is the zenith direction, as shown in Figure 1). The degree of linear polarization is given by

$$
P=-\frac{Q}{I}
$$

where $Q$ and $I$ are the Stokes parameters defined in the customary way [1].

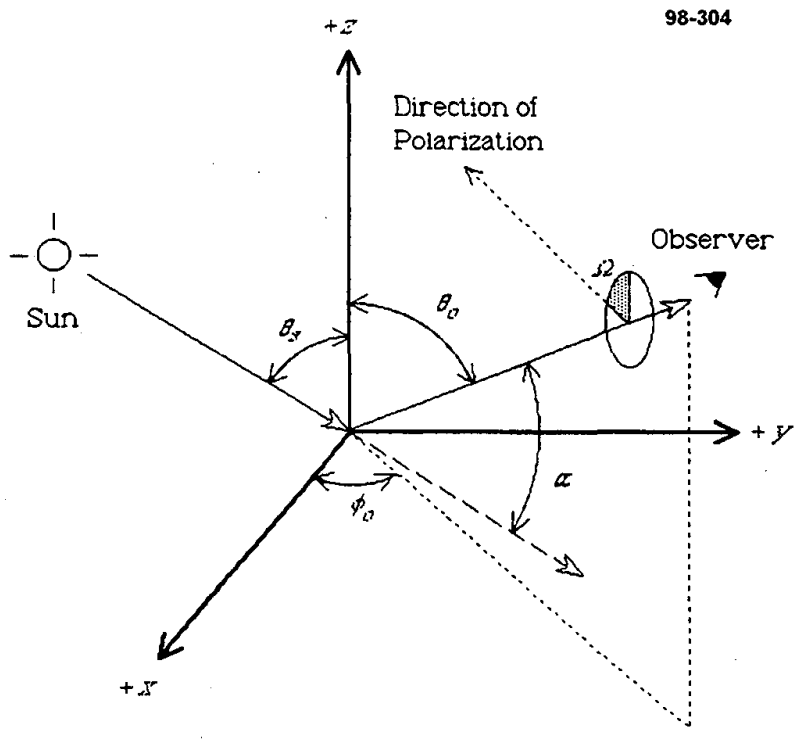

Figure 1 Definition of angles

In general, both $P$ and $\Omega$ depend on all of the following factors:

1. The spatial orientation of the upper surface of the cloud.

2. The phase of the cloud particles.

3. The distribution of the shapes, sizes, and spatial orientations of the cloud particles.

For simplicity, we here restrict our attention to clouds viewed from above (i.e., by high-flying aircraft or satellites) and whose upper surfaces are horizontal. The latter assumption will be a good approximation for stratiform water clouds and most types of ice clouds, such as cirrus and cirrostratus. It is straightforward to generalize our treatment to include cumuliform clouds for which the assumption of horizontal upper surfaces will not be valid. For such clouds, the effects of cloud shadowing will restrict the range of possible directions for the incoming solar radiation and will alter the range of possible directions for the outgoing scattered radiation. In particular, it is often possible to view cumuliform clouds with aircraft flying at altitudes comparable to those of the clouds.

\section{Previous Theoretical Work}

The phase of cloud particles affects $P$ and $\Omega$ through the index of refraction and the absorptive properties of the particles. The real part of the index of refraction differs only slightly between water and ice and is, in any event, easily accounted for in calculations of $P$ and $\Omega$. However, the absorptive properties of the particles substantially complicate the necessary calculations. For ice, in particular, the imaginary part of the index of refraction (which is closely related to the absorption length) varies by about six orders of magnitude in the short-wave infrared (SWIR) and is uncertain at any given wavelength to about a factor of 2 .

For the case of water clouds, the droplets are nearly spherical, so that the problem can be treated according to the theory of Mie scattering which is well developed [2]. The symmetry of the scattering droplets simplifies the polarization properties of the scattered light in some important ways. In particular, $P$ and $\Omega$ depend on $\theta_{\mathrm{S}}, \theta_{0}$ and $\varphi_{0}$ only through the scattering angle, $\alpha$, between the directions of the incoming and scattered rays as defined in Figure 1. Moreover, $\Omega$ is independent of the size distribution of the droplets and is, therefore, a function only of $\alpha$.

The greatest complication for the calculation of $P(\alpha)$ for water clouds arises from the distribution of droplet sizes within the cloud. This distribution varies considerably from one type of cloud to another and is imperfectly known for any type of cloud. There can also be significant variations even within a single cloud.

Some of the best existing calculations of $P(\alpha)$ for water clouds with varying droplet size distributions remain those carried out by Hansen [3] over twenty years ago. Hansen explicitly calculated $P(\alpha)$ for wavelengths, $\lambda$, of 2.25 and $3.1 \mu \mathrm{m}$, but did not present any results for the intermediate or longer wavelengths that would be of interest in the present investigation. However, Hansen's results confirm that the polarization properties of water clouds vary rapidly with wavelength in the SWIR. For example, the primary rainbow (corresponding to enhanced back-scattered intensity and polarization at $\alpha \cong 135^{\circ}$ ) is a prominent feature at $\lambda=2.25 \mu \mathrm{m}$ but has completely disappeared at $\lambda=3.1$ $\mu \mathrm{m}$, owing to the high degree of absorption within the cloud droplets for wavelengths near the center of the $3 \mu \mathrm{m}$ band. Due to the rapid variation in the degree of polarization with wavelength in the vicinity of the $3 \mu \mathrm{m}$ band, an interpolation of Hansen's results at the bracketing wavelengths, or an extrapolation to longer wavelengths, could not be expected to yield reliable values for $P(\alpha)$.

The calculation of both $P$ and $\Omega$ is much more complicated for ice clouds than for water clouds, since the ice crystals comprising the clouds can take on a variety of shapes. The basic symmetry of ice crystals is hexagonal but, within this constraint, a wide variety of shapes are possible, including plates, columns, and double pyramids, all with a wide range of possible ratios among the principal axes $[4,5,6]$. Individual crystals may also fuse together in a wide and 

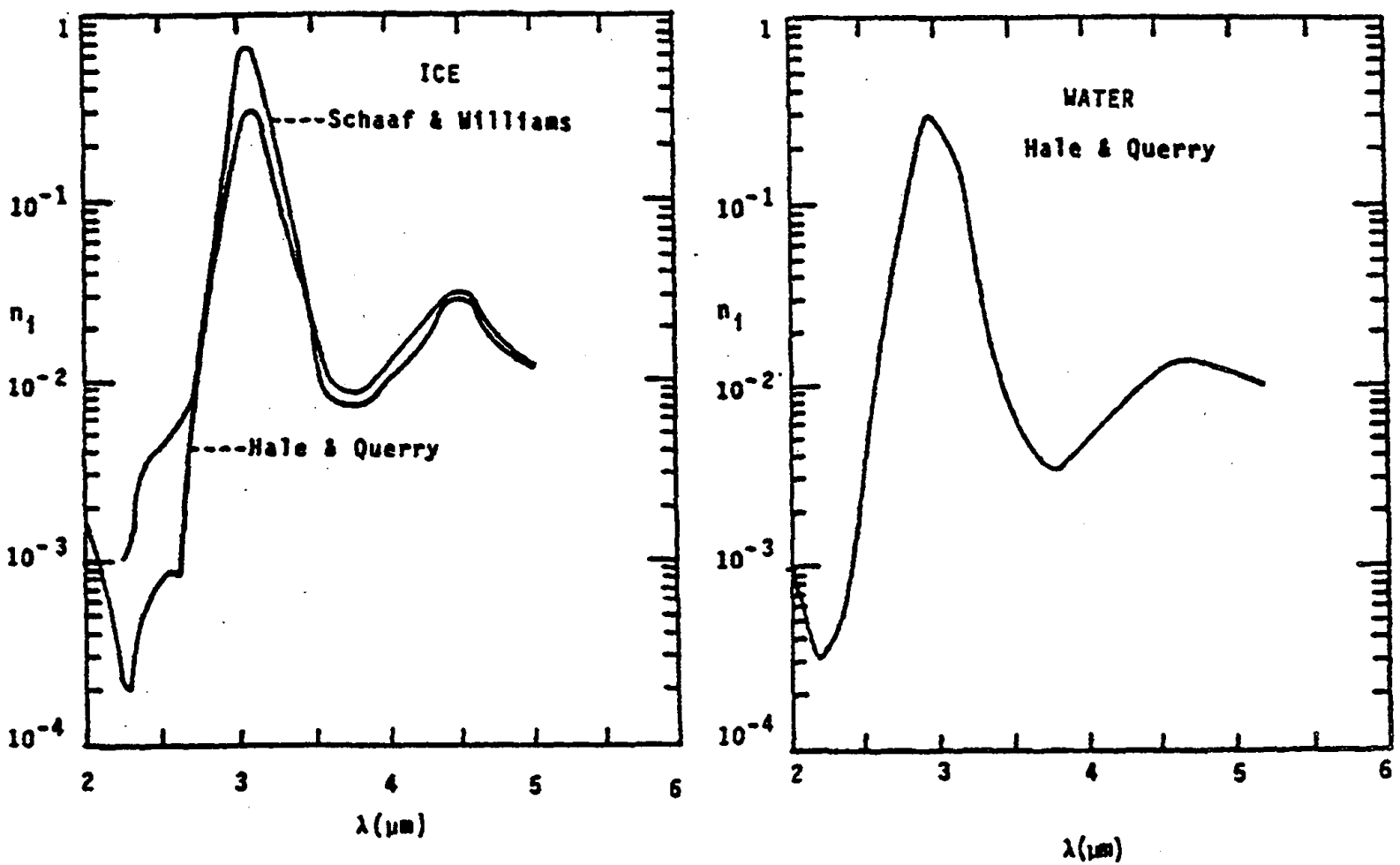

Figure 2 The imaginary part of the index of refraction for water and ice as a function of wavelength $[8,9]$

complex variety of ways. A given cloud may thus be composed of individual crystals and/or crystal clusters with a variety of shapes and a range of sizes and spatial orientations for each shape.

As a consequence of these complications, $\Omega$ is no longer a unique function of $\theta_{S}, \theta_{0}$, and $\varphi_{0}$, and, even for a specified distribution of crystal shapes, sizes, and orientations; $P$ is no longer a function of $\alpha$ alone. Nevertheless, those computational results that exist for the degree of polarization of radiation scattered from ice clouds are generally averaged appropriately over $\theta_{\mathrm{s}}, \theta_{0}$, and $\varphi_{0}$ and presented as functions of $\alpha$ only [6,7]. Nearly all existing calculations of $P$ for ice clouds apply to visual wavelengths only. A few results are available for SWIR wavelengths and provide encouraging evidence that measurements at such wavelengths can, indeed, yield useful information about the nature of the cloud particles.

\section{Theoretical Approach}

Even in the simplest cases, the treatment of polarization of radiation scattered from clouds requires extensive numerical computations. Two techniques have been developed for such computations: the "doubling method" [3] and Monte Carlo techniques [2].

While large fractional polarization occurs for certain scattering geometries for single scattering, the randomizing effect of multiple scattering will significantly reduce $P$. Thus one objective is to maximize the contribution of singly scattered photons in the measured signal. One way to do this is to choose a wavelength at which the scattering particles are highly absorbing.

As noted in the previous section, the imaginary component of the index of refraction, $n_{i}$, of a substance is a direct measure of how strongly that substance absorbs electromagnetic radiation. Figure 2 illustrates the variation of $n_{i}$ for water and ice as a function wavelength, $\lambda$, from 2 to $6 \mu \mathrm{m}$, as measured experimentally by Hale and Querry [8] and by Schaaf and Williams [9]. Note that for both water and ice, the maximum values of $n_{i}$ occur in the range of about 2.8 to $3.3 \mu \mathrm{m}$, with a more subdued peak of moderately high values centered near $4.8 \mu \mathrm{m}$.

Once it enters the cloud top, a light ray may be scattered by a water droplet or ice particle, or it may be absorbed by such a particle or by the water vapor in the saturated air between particles. Note, however, that the SWIR absorption band for water vapor is at somewhat shorter wavelengths, about 2.6 to $2.8 \mu \mathrm{m}$, than the absorption bands for liquid water or solid ice.

Let $r$ be the ratio, per particle-light ray interaction, of the probability of scattering to the probability of absorption 


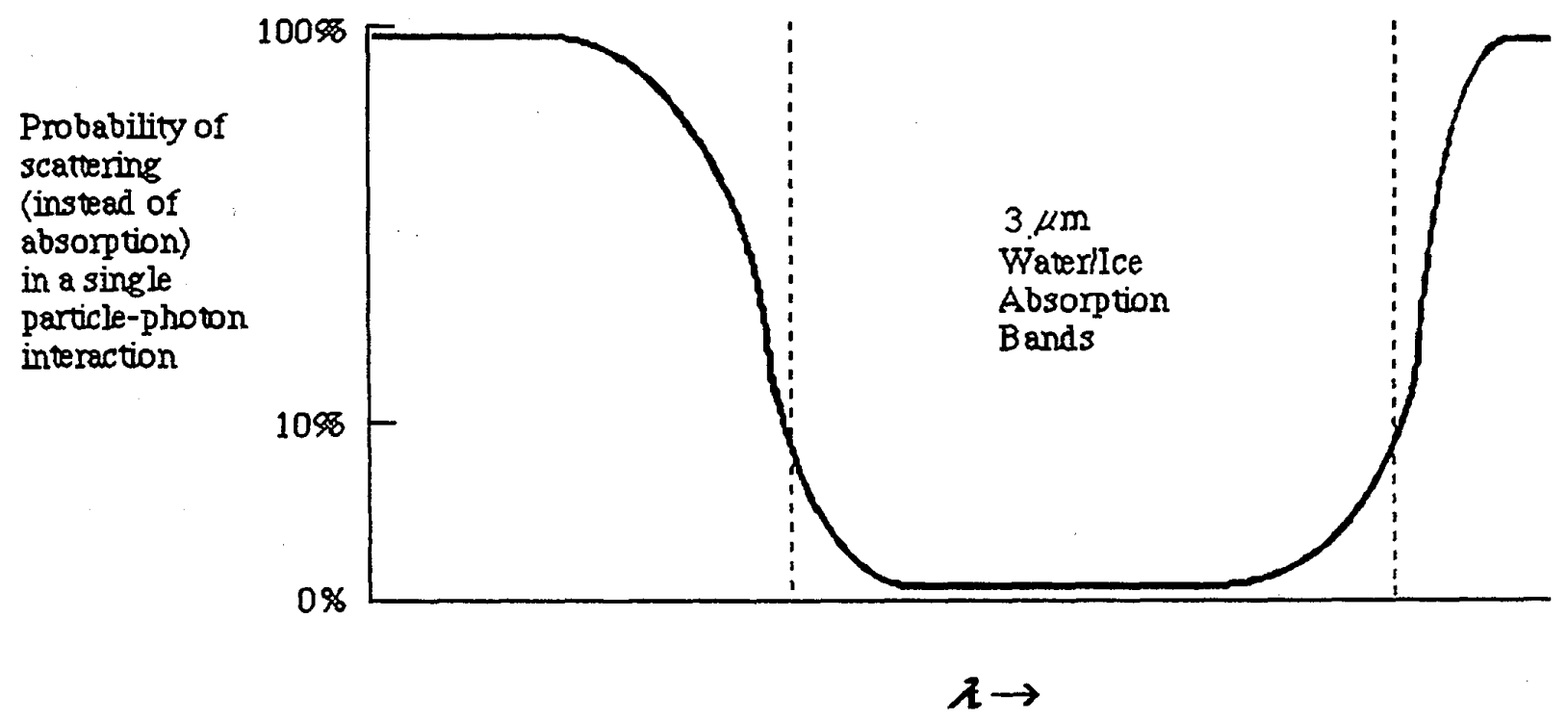

Figure 3 Schematic of the scattering probability near the water/ice absorption band

(either by the particle or by the intervening water vapor) along the path traversed by the ray since entering the cloud top or since the previous interaction of the ray with a particle. The crucial measurement band, as shown schematically as shown in Figure 3, is to be chosen such that $r$ is less than about $10 \%$ throughout the band. The permissible values for the wavelength range of this band will depend on the cloud properties, including the size distribution of the cloud particles, as well as the pressure and temperature at the cloud top, which determine the density of water vapor in the saturated air. For the purpose of this discussion, we make the simplifying assumption that for a given cloud, $r$ is a function only of wavelength.

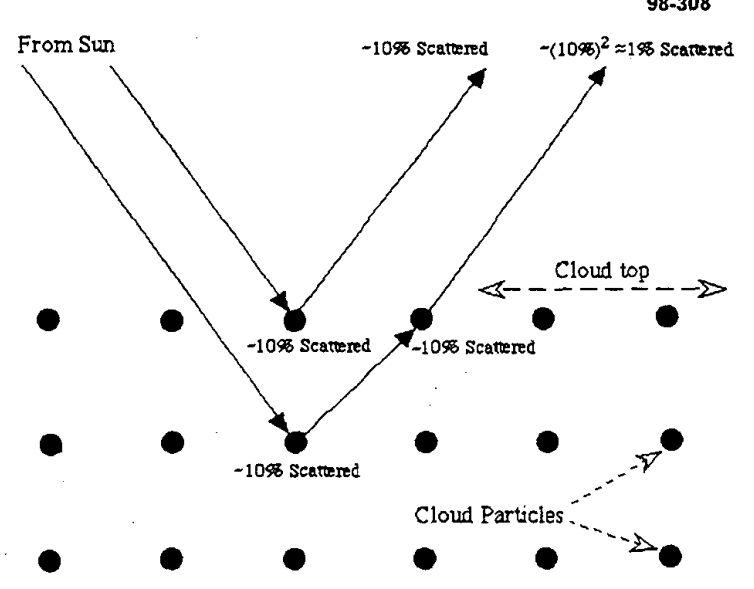

Figure 4 Schematic of the scattering within a cloud

While the amount of scattered radiation in this band will be more than adequate for the measurement of $P$ and $\Omega$, the fraction of light rays that are doubly (or multiply) scattered and still escape from the cloud top without being absorbed will be of the order of $(10 \%)^{2}$, or about $1 \%$. As a result the percentage of the radiation reaching the detector that has undergone more than one scattering event will be $\sim(10 \%)^{2} / 10 \%$ or about $10 \%$, as shown schematically in Figure 4 . Thus, the great majority of the rays reaching the detector will have undergone only a single scattering at the cloud top, so that the light reaching the detector will generally have a high degree of linear polarization.

It is also of interest to determine $P$ and $\Omega$ near the $3 \mu \mathrm{m}$ band, where $r$ is much greater than 1 , in order to compare the measured results with theoretical expectations for rays that are multiply scattered at the cloud top. Similarly of interest is the determination of $P$ and $\Omega$ for measurement bands wherein $r$ is much less than $10 \%$ throughout the band in order to determine the polarizing effects of Rayleigh scattering by atmospheric molecules and Mie scattering by aerosols above the cloud top and to compare these results with theoretical expectations $[10,11]$.

\section{Simulation}

In order to develop a complete theoretical understanding of the behavior of $P$ and $\Omega$ as functions of $\theta_{\mathrm{S}}, \theta_{0}, \varphi_{0}$ as well as particle size, shape, and orientation distributions for water droplets, ice crystals, and mixtures of the two, we have initiated an extensive new set of numerical Monte Carlo calculations. These calculations are being performed at the Maui High Performance Computing Center (MHPCC), a supercomputing facility with a total computational power in excess of 130 gigaflops.

The codes necessary to carry out these calculations are presently under development. In order to achieve the 
highest possible level of confidence in our results, we are carrying out the numerical calculations in two different ways using two completely independent codes. In the first technique, each ray is propagated from the Sun to the cloud top and is then either absorbed or scattered randomly, with a suitable probability distribution, into a direction from which it may be either scattered again by another cloud particle or detected with an air- or satellite-based instrument. In the second method, the calculations are carried out in a timereversed fashion. Each incoming ray is propagated backward from the detector to the cloud top and then scattered randomly, with a suitable probability distribution and with an appropriate amplification factor to allow for the possibility of absorption, into a direction from which the incoming ray would have been either scattered previously by another cloud particle or propagated downward from the Sun.

In both techniques, we allow for the possibility of either single or multiple scattering, both within the cloud and in the clear air (Rayleigh scattering by atmospheric molecules and Mie scattering by aerosols) along the line of sight from the radiation source to the cloud top and from the cloud top to the detector. For adequate statistics, and in light of the number of parameters in the calculation (incoming and outgoing directions of propagation, as well as the cloud particle phase, size, shape, and orientation distributions), each of these techniques requires that we calculate the propagation of $-10^{8}$ rays. Calculations of this magnitude are well within the capabilities of the MHPCC.

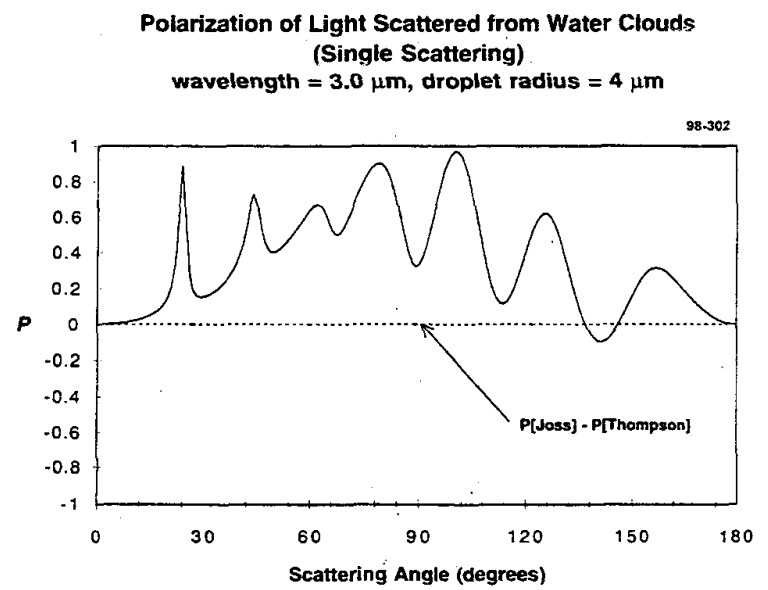

Figure 5 Sample of computer model predictions

We have developed and used a Monte Carlo code that employs the first of these two methods to calculate the polarization of sunlight scattered from a water cloud, under the simplifying assumptions of single scattering for all scattered rays and a $\delta$-function distribution of droplet radii (i.e., a uniform droplet radius of $4 \mu \mathrm{m}$ ). We have carried out calculations for the full range of possible scattering angies $\left(0^{\circ} \leq \alpha \leq 180^{\circ}\right)$ and for three SWIR wavelengths of interest $(2.5,3.0$, and $3.5 \mu \mathrm{m}$.) Our results for $P$ corresponding to $\lambda=3.0 \mu \mathrm{m}$ are displayed in Figure 5 .

In order to verify the accuracy and reliability of these results (and to validate our Monte Carlo code), we have compared them with independent calculations carried out by Thompson [12] using a completely different code that employs different numerical techniques. In all cases our results for $P$ and $\Omega$ differed from Thompson's computations by less than one part in $10^{6}$. The dashed line on Figure 5 represents the difference between our results for $P$ and those obtained by Thompson. For all values of $\alpha$, this line cannot be distinguished from zero on the scale of the figure.

\section{INSTRUMENTATION}

To make measurements for this program, we wanted to make use of existing assests to minimize development time and costs. The FISTA (Flying Infrared Signatures Technology Aircraft) aircraft, which we have used in past investigations, was an obvious choice. The FISTA aircraft, shown in Figure 6, is a modified NKC-135E aerial refueling tanker (serial number 55-3135) built by Boeing that is based with the $452^{\text {nd }}$ Test Squadron at Edwards AFB, California. It has a continuous flying time of 11 hours, a maximum range of about $11,000 \mathrm{~km}$, and a maximum ceiling of 13,000 meters.

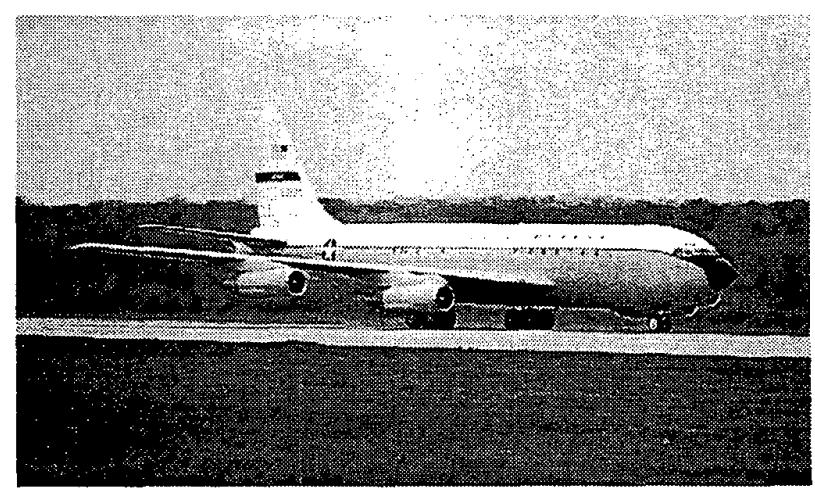

Figure 6 FISTA aircraft

FISTA has been modified to act as a test bed for a wide variety of infrared and visible sensor systems. It has 20 windows on the starboard side of the fuselage with nearly all consisting of a $31.8 \mathrm{~cm}$ diameter clear aperture which are filled by a clear $1.9 \mathrm{~cm}$ thick Lexan window, an instrument window or periscope. The instruments, which can be run simultaneously, are mounted at these windows and look to the right during flight. Provisions also exist to mount periscopes in selected windows which allow the instrument to look in a variety of directions including fore, aft, up, and down.

The aircraft is also equipped with a GPS (Global Positioning System) to provide data on the position, heading and speed of the aircraft as well as its attitude. All this data 
along with a GPS-derived time base are recorded for later use in interpreting the data and calculating scattering angles.

\section{Polarization Measurement Technique}

The method used to measure the degree of polarization, $P$, and the polarization angle, $\Omega$, was to make a series of three successive measurements through polarization filters with orientations at $0^{\circ}, 60^{\circ}$, and $120^{\circ}$ with respect to the instruments' frame of reference. This results in a "triplet" of polarization measurements or images designated $I_{1}, I_{2}$, and $I_{3}$. This triplet can be converted into the total intensity (i.e. the sum of the polarized and unpolarized light intensities), $I_{T O T}$, as follows:

$$
I_{\text {TOT }}=\frac{2}{3}\left(I_{1}+I_{2}+I_{3}\right)
$$

The polarized intensity, $I_{\mathrm{P}}$, can be calculated as follows:

$$
I_{P}=\frac{4}{3} \sqrt{I_{1}^{2}+I_{2}^{2}+I_{3}^{2}-I_{1} I_{2}-I_{1} I_{3}-I_{2} I_{3}}
$$

The degree of polarization, $P$, is then:

$$
P= \pm \frac{I_{P}}{I_{T O T}}
$$

The sign of $P$ is determined by the polarization angle $\Omega$. $P$ is positive when $0^{\circ} \# \Omega \# 45^{\circ}$ and negative when $45^{\circ} \# \Omega \# 90^{\circ}$.

The angle that the polarization vector of the incoming radiation makes with the instruments' frame of reference, $\omega$, can be calculated using the following relation:

$$
\omega=\cos ^{-1}\left(\sqrt{\frac{I_{1}-\frac{1}{2}\left(I_{T O T}-I_{P}\right)}{I_{P}}}\right)
$$

This angle, $\omega$, can then be converted to the polarization angle, $\Omega$, by the appropriate transformation from the coordinate system of the instrument to the defined Sunscattering point-observer reference frame. In the analysis presented in this paper, we have confined our analysis to determining only the absolute value of $P$. We will address the question of the polarization angle, $\Omega$, in the future.

\section{Experiment Plan}

Typically the FISTA aircraft is limited to fly within an area with a $1,900 \mathrm{~km}$ radius (depending on weather conditions) centered on Edwards AFB. The specific flight path for a mission depends on an assessment of meteorological satellite weather maps from the day prior to the flight in order to identify promising sites to make observations.
In order to maximize the range of scattering angles sampled, observations were confined to times near local sunrise or sunset when the Sun was its closest to the horizon. In order to sample a cloud deck or feature through a large range of scattering angles, the aircraft would make circular orbits (with a constant bank angle in the $5^{\circ}$ to $45^{\circ}$ range) around a particular group of clouds so that they could be observed under various lighting conditions. During a typical flight, the aircraft would make a half a dozen or more such orbits over interesting targets as well as some additional observation during level flight when traveling from one target to another.

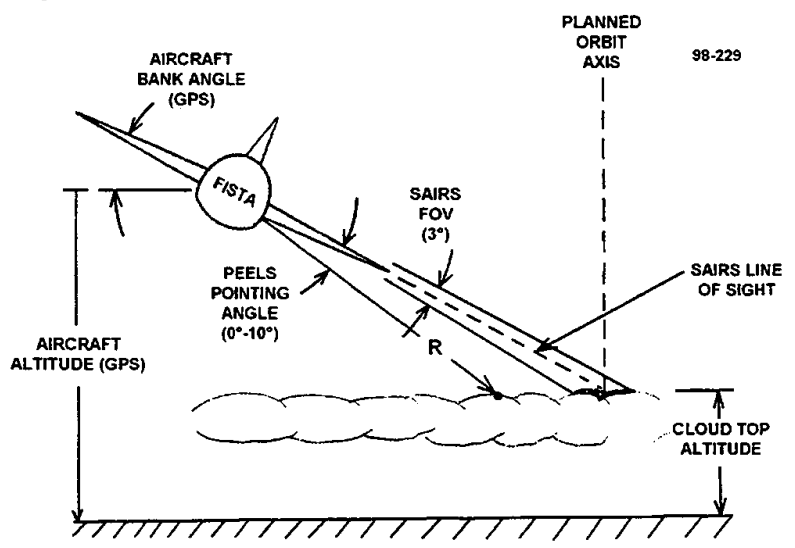

Figure 7 FISTA instrument viewing and orbit geometry

The instruments carried by FISTA were typically pointed in such a fashion that they would observe approximately the same part of the cloud deck throughout an orbit as shown in Figure 7. In practice the aircraft inevitably drifted in relation to the clouds (due to upper atmospheric winds) and it was impractical to track a single feature for an entire orbit even when an instrument was being pointed manually. Still, the results would prove useful so long as the cloud deck being observed was relatively homogeneous or any changes in scattering properties tracked.

While several instruments were flown during the 1997 FISTA flights, the following successfully provided data that proved to be useful in our investigations.

\section{Michelson Interferometers}

For the 1997 FISTA flights, the aircraft was equipped with a trio of Michelson interferometers supplied by AFRL ( $A$ ir Force Research Laboratory). Originally designed to provide spectra with complementary properties in overlapping spectral regions ranging from the SWIR to M/LWIR, these instruments were modified with the addition of three polarization filters (with the orientation of each offset by $60^{\circ}$ from its neighbors) to provide information on the degree of polarization and its angle ( $P$ and $\Omega$ respectively) as a function of wavelength, $\lambda$. It was hoped that we could obtain polarization data in the spectral regions adjacent to some of the infrared absorption bands of water. 
Interferometer 102 makes use of an InAs detector to produce spectra in the 1.5 to $3.1 \mu \mathrm{m}$ range with a resolution of $4 \mathrm{~cm}^{-1}$. It produces one spectrum each 1.1 second with the filter wheel being advanced after each scan. As a result, 3.3 seconds are required to obtain the measurements needed to define $P$ and $\Omega$. The instruments field of view is approximately $1.5^{\circ}$ in diameter.

Interferometer 103 uses a $\mathrm{HgCdTe}$ detector to produce spectra in the 2 to $7 \mu \mathrm{m}$ range with a resolution of about 1 $\mathrm{cm}^{-1}$. This instrument produces one spectrum each second and takes 3 seconds to make the measurements required to define the polarization properties of the scene. It has a diamond-shaped field of view with each side being about $0.5^{\circ}$ across.

Interferometer 105 uses an InSb detector to make spectral measurements in the 2.0 to $5.5 \mu \mathrm{m}$ range with a resolution of $1 \mathrm{~cm}^{-1}$. Like Interferometer 103, it makes one spectrum each second and requires 3 seconds to obtain data through its trio of polarization filters. Unlike the other interferometers which were flown with polarization filters for all of the 1997 FISTA flights, Interferometer 105 was equipped with polarization filters for only the September flights. The instrument's field of view is about $1^{\circ}$ in diameter.

Raw spectral data from these instruments are recorded digitally along with measurement of calibration sources for post-flight processing. All three Michelson interferometers are equipped with their own dedicated video camera coaligned with each interferometer's field of view to provide a record of what was observed at any given time.

Because of the relatively narrow field of view of each of these instruments and the $3+$ second time period required to make the complete polarization measurements, these instruments had to manually track a succession of cloud feature targets to compensate for the aircraft's motion. Since there was no way to precisely determine the pointing angles of the instruments relative to the aircraft's frame of reference during these maneuvers, the scattering angles for these data could only be crudely estimated. This presented no major problem for these initial flights since we were interested in assessing these instruments ability to detect any wavelength dependent effects of polarization.

\section{SAIRS}

SAIRS (Schottky Array InfraRed Sensor), also supplied by AFRL, is capable of producing images at wavelengths between about 1 and $5 \mu \mathrm{m}$. For these flights, SAIRS was equipped with a spectral filter to limit its bandpass to 2.33 to $2.65 \mu \mathrm{m}$ in the SWIR. This corresponds roughly to the shoulder of the water absorption band centered at $3 \mu \mathrm{m}$.

This instrument's detector consists of a $160 \times 244$ element PtSi array with a $3^{\circ} \times 5^{\circ}$ field of view that is readout at video rates (i.e. $30 \mathrm{~Hz}$ ). An infrared polarizing filter was attached to the instrument which rotated through $60^{\circ}$ in discrete steps every 2 seconds. As a result, 2 to 4 seconds were required (depending on the phasing between video images, the filters, and post-flight frame grabbing) to obtain the triplet of polarization measurements needed to determine the degree of polarization and its angle $(P$ and $\Omega$ respectively). SAIRS data were recorded on videotape and selected images were then digitized and calibrated after the flight for this investigation.

During the 1997 FISTA flights SAIRS was targeted in two ways. Like the Michelson interferometers, SAIRS could be manually moved during an observation run to track specific targets in an effort to detect any polarization effects. As with the interferometers, there was no way to precisely determine the pointing angles of the instruments relative to the aircraft's frame of reference during these periods of manual tracking. As a result, the scattering angles for these data could only be crudely estimated.

Data could also be taken with the instrument locked in position and pointing approximately at the central axis of the aircraft's circular orbit as shown in Figure 7. This corresponded roughly to a pointing position at a right angle to the aircraft's direction of travel and level with its frame of reference. Scattering angles could be confidently determined during these times to an accuracy of a couple of degrees.

\section{MAVIS}

MAVIS (Multispectral Airborne Video Imaging System), built by Visidyne, Inc., is a multispectral video camera operating at visible wavelengths. Although this instrument is unable to observe at any of the infrared wavelengths that correspond to the water absorption bands that are of interest to this investigation, it does supply polarization data that complements that obtained in the infrared. It also offers us the ability to further assess the scattering properties of the clouds being observed independent of the effects we seek in the infrared.

MAVIS is based on the Xybion Electronic System Corp. IMC-301W intensified CCD camera. It consists principally of an $18 \mathrm{~mm}$ diameter microchannel plate intensifier coupled to a high-resolution $2 / 3$ inch-format $(17 \mathrm{~mm}$ format) $\mathrm{CCD}$ imager via an optical fiber coupling. The $\mathrm{CCD}$ has an imbedded microprocessor for precision control of the array's gain and exposure. The intensifier's gain can be controlled to offer a gain of 100 to 70,000 and can be gated to provide exposure times as short as 10 nanoseconds.

The instrument is equipped with a precision balanced filter wheel that rotates at $300 \mathrm{rpm}$. With its six filter positions whose change is synchronized with the camera's video signal, it can obtain sequential filtered images at video rates. For these investigations, the first three filter wheel positions were occupied by blue, green, and red filters to provide color information on the scene being observed. The 
remaining three positions were occupied by polarization filters with orientations sequentially offset by $60^{\circ}$. This filter set allowed a triplet of polarization measurements to be taken in about 67 milliseconds. This made it possible for the degree of polarization and its angle $(P$ and $\Omega$ respectively) to be determined at visible wavelength band ranging from 0.4 to $0.7 \mu \mathrm{m}$.

Data from MAVIS are recorded on videotape with selected frames subsequently digitized and calibrated after the flight. The calibrated portion of the images consists of $461 \times 388$ pixel region in the center of the image. The instrument is equipped with a standard $\mathrm{C}$-mount so that a variety of objective lenses can be used with a field of view tailored to the requirements of the flight. For example, for Flight 9719 the effective field of view of the calibrated MAVIS image was about $7.0^{\circ} \times 5.5^{\circ}$ while on Flight 9721 it was $10.4^{\circ} \times$ $8.3^{\circ}$.

\section{PEELS}

The last instrument that yielded useful data during the 1997 FISTA flights was PEELS (Portable Eyesafe Environmental Lidar System) also built by Visidyne, Inc. PEELS made lidar soundings of the environment being observed, thus complimenting the observations of the other instruments.

Originally PEELS was designed as a portable, tripodmounted lidar system meant to obtain range and depolarization measurements of various types of clouds and aerosols within its nominal $25 \mathrm{~km}$ range. Because of its design and the wavelength employed, it has been rated as eyesafe and skinsafe. For the 1997 FISTA flights PEELS was modified to operate using a single optical aperture (as opposed to the separate transmitting and receiving optics it normally uses) and repackaged to conform to the aircraft's instrument interface standards.

The transmitter for PEELS is a Nd:YAG pulsed laser with a KTP OPO operating at a wavelength of $1.574 \mu \mathrm{m}$. For the FISTA flights, the output beam was vertically polarized. The typical laser pulse has an energy of 45 millijoules and a width of 10 nanoseconds. The maximum repetition rate for the laser is $10 \mathrm{~Hz}$ and the nominal beam divergence is 0.5 milliradians. The receiver on PEELS is a commercially available $20-\mathrm{cm}$ Schmidt-Cassegrain telescope with a pair of InGaAs detectors fitted with polarizing filters. Its field of view is about 1 milliradian.

The range resolution of the system is variable and can be as small as 8 meters. For these FISTA observations, resolution was set at 150 meters and as many as 100 soundings were averaged to yield each measurement.

PEELS performs measurements of range and backscatter depolarization only. The range measurement is based on the round trip time of the laser pulse. The percent depolarization is measured by taking the ratio between the horizontal polarization component of the return signal and the sum of the vertical and horizontal polarization return signals. This quantity provides an independent method for determining the composition of the clouds being observed [13].

PEELS was mounted in such a way that it could be aimed at elevation angles ranging from $0^{\circ}$ to $-10^{\circ}$ with respect to the aircraft's frame of reference. When FISTA was in a banking turn, as was typical during an orbit, this offset further reduced the range to the clouds being observed thus increasing the backscatter signal. This adjustable elevation angle offset also allowed the instrument to obtain measurements during level flight.

\section{OBSERVATIONS}

The 1997 flights can be broken down into two groups. The first consisted of three flights conducted on July 17, 18, and 22 (designated Flights 9714, 9715, and 9716 respectively). These flights were designed to check the modifications to the instruments and other systems. Approximately six hours of data were gathered during these "dry run" flights. The results indicated that the instruments were functioning adequately and that the modified instruments were yielding some useful polarization data.

The second group of flights on September 18, 19 and 20, 1997 (designated Flights 9719,9720 , and 9721 respectively) built on the experience from the first group of flights. They successfully collected ten hours of data over various locations scattered across the western United States.

Because of the enormous amounts of data, efforts were made to identify observations that included clouded regions which displayed a large degree of polarization. As a result of our review of the raw data, we focused our analysis efforts on data obtained during Orbit 6 of Flight 9719 flown on September 18, 1997. Particularly interesting data obtained during Orbits 3 and 7 during Flight 9721 flown on September 20, 1997 were analyzed in a more limited fashion. Data from Orbit 3 of this flight included interesting observations of Sun glints.

\section{Scene Description}

Orbit 6 of Flight 9719 took place over the CaliforniaNevada border on September 18, 1997 from approximately 17:11 to 17:20 UT. An infrared GOES meteorological satellite image from 17:00 UT, shown in Figure 8, gives a synoptic view of the cloud system observed. Imagery from this orbit showed an unbroken deck of stratus clouds with the visible top of the deck grading from a puffy cumuluslike to a more cirrus-like appearance. Lidar measurements by PEELS indicated that the cloud tops were at a fairly uniform height of about $8 \mathrm{~km}$ or about $3 \mathrm{~km}$ below the aircraft. The air temperature was estimated to be about -43 C. 
Lidar measurements for this orbit shown in Figure 9 show that there is much variability in the return signal and percent depolarization as a function of time and altitude throughout the orbit. Because of this variability and PEELS' $-10^{\circ}$ elevation angle offset from the other instruments during this orbit, this data can not generally be used directly with information from the other instruments to sort out what has been observed and when. Still, the data can be used to characterize the general properties of the cloud deck throughout the orbit.

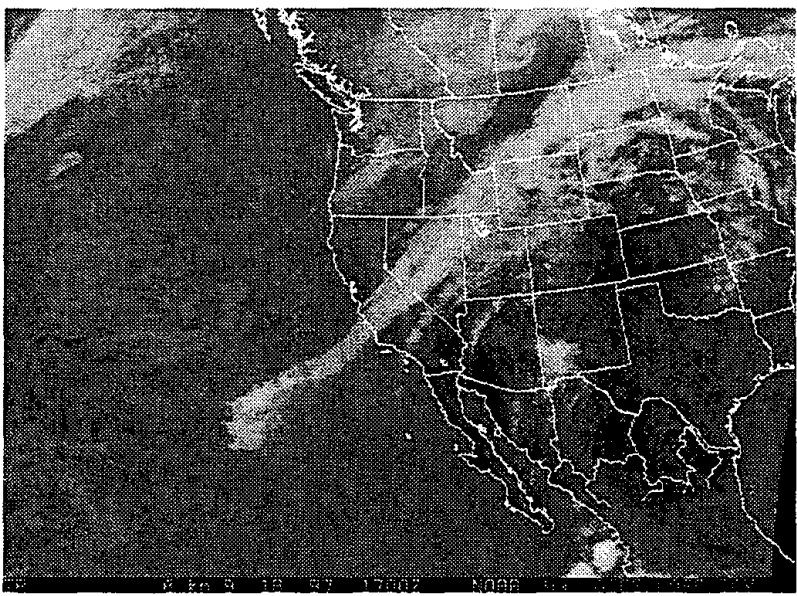

Figure 8 GOES IR image near time of Flight 9719, Orbit 6

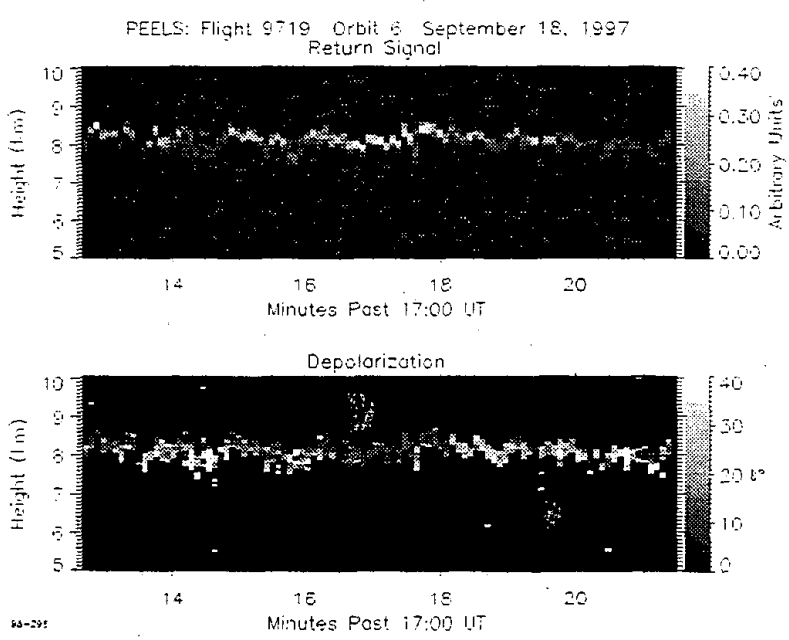

Figure 9 PEELS data for Flight 9719, Orbit 6

The relatively large return signal depolarization typical for much of this orbit is consistent with the presence of ice in the clouds. Between approximately $17: 15$ and 17:18 UT the amount of depolarization is less than that expected for an ice cloud (which typically is greater than $20 \%$ ) but more than what has been observed in water clouds (which is in the $1 \%$ to $4 \%$ range) [13]. This could indicate the presence of a water-ice mixture in parts of the cloud deck.

Orbits 3 and 7 of Flight 9721 took place over north-central Colorado on September 20, 1997 from approximately 13:51 to $13: 58$ UT and $14: 42$ to $14: 47$ UT respectively. An infrared GOES meteorological satellite image from 14:00 UT, shown in Figure 10, gives a synoptic view of the cloud system observed. Imagery and an analysis of the PEELS data for Orbit 7 (which is typical for this whole flight) shows a thick deck of cirrus-like clouds at an altitude of 9 to $9.5 \mathrm{~km}$ or about 1.5 to $2 \mathrm{~km}$ below the aircraft. The air temperature was estimated to be about $-45 \mathrm{C}$.

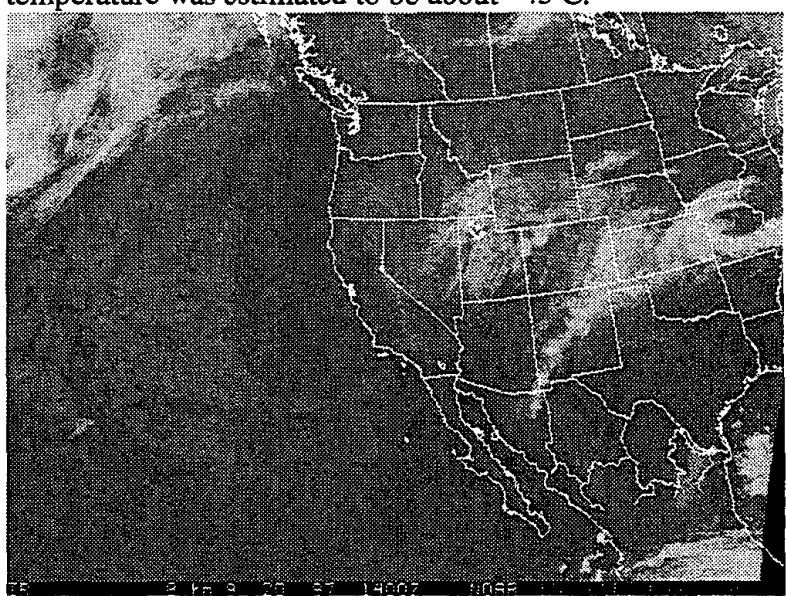

Figure 10 GOES IR image near time of Flight 9721, Orbit 3
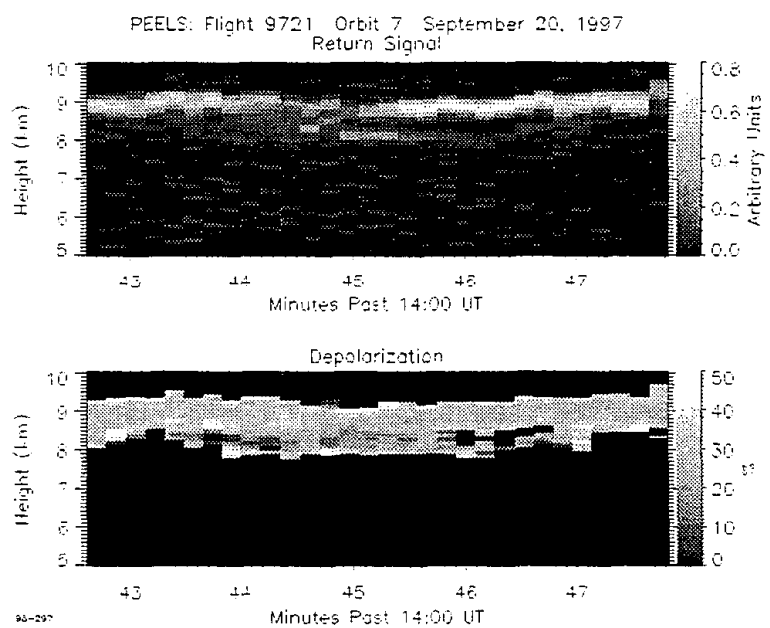

Figure 11 PEELS data for Flight 9721, Orbit 7

Processed PEELS data for Flight 9721, Orbit 7 are shown in Figure 11. Unlike the previous data set, the clouds seem to be much more uniform. Interestingly there is a useful return signal over a thickness of about $1.3 \mathrm{~km}$ and hints of a second, slightly denser cloud layer at an altitude of about $8.3 \mathrm{~km}$. This deck of clouds seems to be optically thinner than the other cloud deck observed allowing light to penetrate deeply before being scattered. The $21 \%$ to $28 \%$ depolarization PEELS observed during this orbit is consistent with the presence of ice throughout this cloud deck. 


\section{Infrared Wavelength Results}

For the 1997 flights, the two instruments used to obtain polarization measurements in the infrared were the trio of Michelson interferometers and the SAIRS imager. Because of the low signal strength in and near the absorption bands of interest in the infrared, the polarization measurements made by the interferometers are dominated by noise. As a result, these instruments could not provide any useful polarization results in the SWIR to $M / L W I R$ wavelength bands as originally hoped.

Despite this, the interferometers did provide spectra that were able to provide additional insights into the composition and physical state of the clouds being observed by the other instruments. A typical spectrum for water and ice clouds obtained by Interferometer 102 during Flight 9715 shown in Figure 12 illustrates the difference seen in the spectra of such clouds.

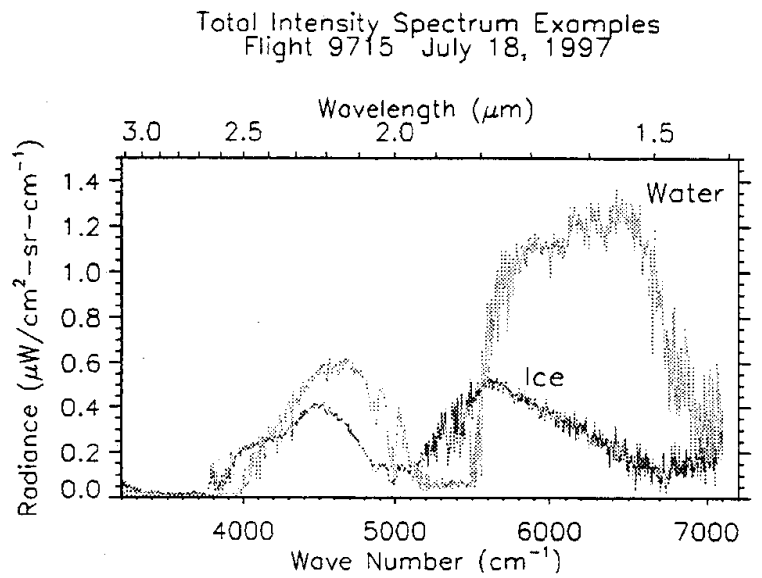

Figure 12 Example of spectra for water and ice clouds from Interferometer 102

A spectrum from the same instrument from Orbit 6 of Flight 9719 is shown in Figure 13. Its shape, which changed little throughout the orbit, indicates that water ice predominates in general agreement with the results obtained by PEELS. An averaged spectrum obtained during Orbit 7 of Flight 9721 shown in Figure 14 displayed similar properties indicating that ice is present.

While the Michelson interferometers were unable to provide any usable polarization data during this series of flights, the SAIRS imager did yield very interesting results in the SWIR. Data from this instrument were processed using two distinctly different methods. The first and most obvious method was to register a polarized-filter image triplet from the instrument and calculate the total intensity and degree of polarization on a pixel by pixel basis. This would yield a set of images giving the total intensity and percent polarization in various parts of the scene.

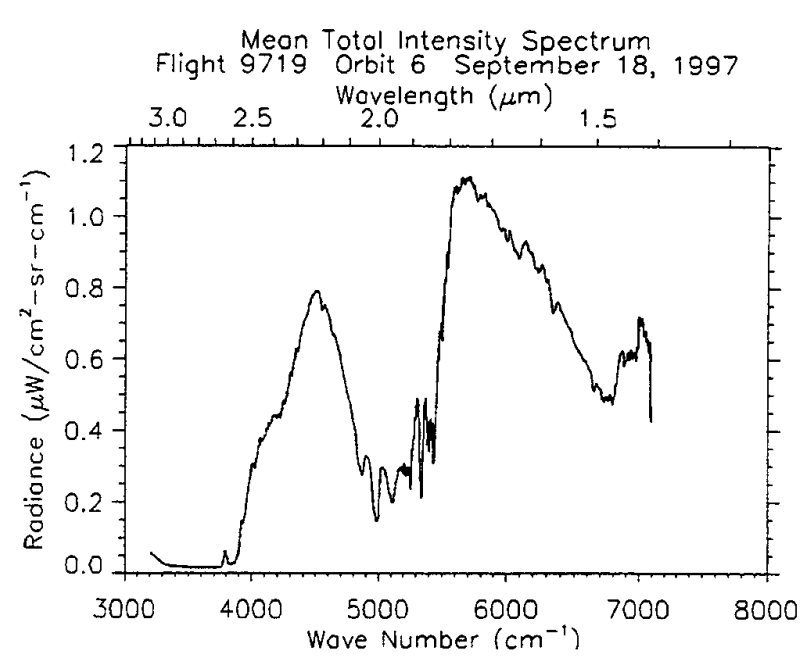

Figure 13 Average spectrum from Flight 9719, Orbit 6

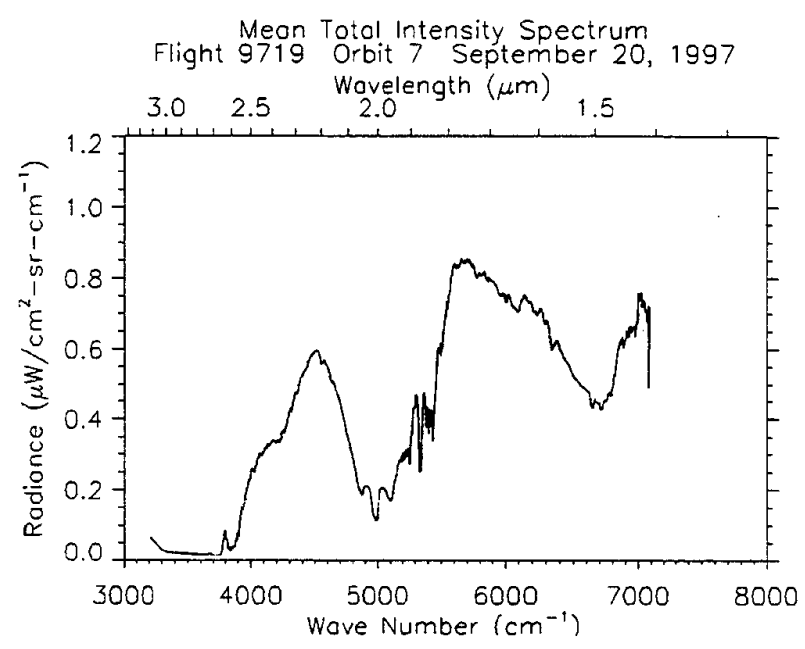

Figure 14 Average spectrum for Flight 9721, Orbit 7

Due to the 2 to 4 second period needed to change the polarization filters three times on SAIRS and its relatively narrow field of view, parallax effects were quite noticeable in the polarized filtered image triplets. As a result, features in the foreground moved more than features further away during the time to make the required observations. This property made it impossible to register the three images by simply translating them.

In order to partially correct for these parallax effects and register the images in a triplet, each row of pixels in a SAIRS image was shifted by an amount that was proportional to its row number. This technique works best with a cloud deck that displays little vertical relief. An example of such a registered total intensity image is shown in Figure 15 taken at about 17:15:35 UT. The attempt to correct for parallax has resulted in the slanted border on the right side of the image. The irregular shaped edge in the lower left corner of the image is where an alphanumeric display containing the time and other engineering data 
appeared in the original images that was removed before processing.

The corresponding degree of polarization image in Figure 16 has a mean scattering angle of $143^{\circ}$. While some of the high percent polarization values near the boundaries between light and dark portions of the image are artifacts produced by residual misregistration of the images, it does present a reasonable representation of the polarization in the scene.

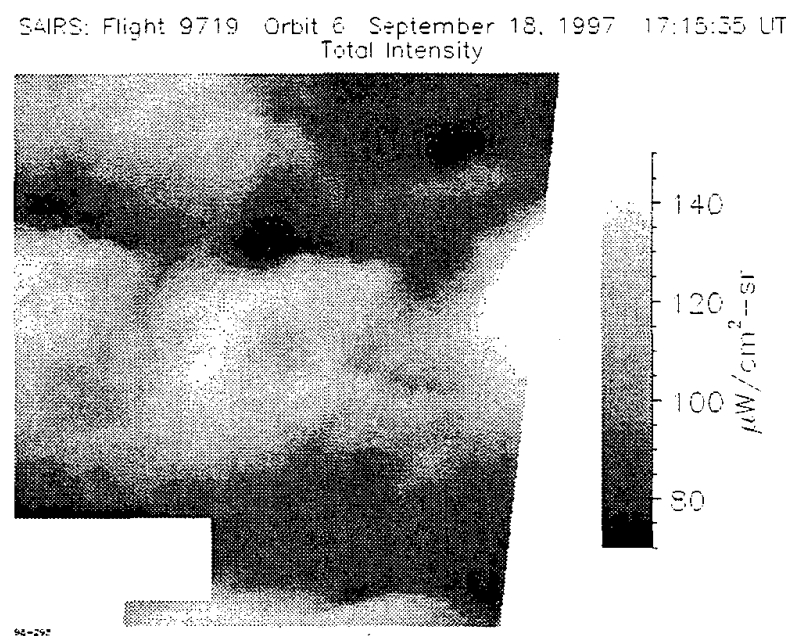

Figure 15 Sample SAIRS total intensity image

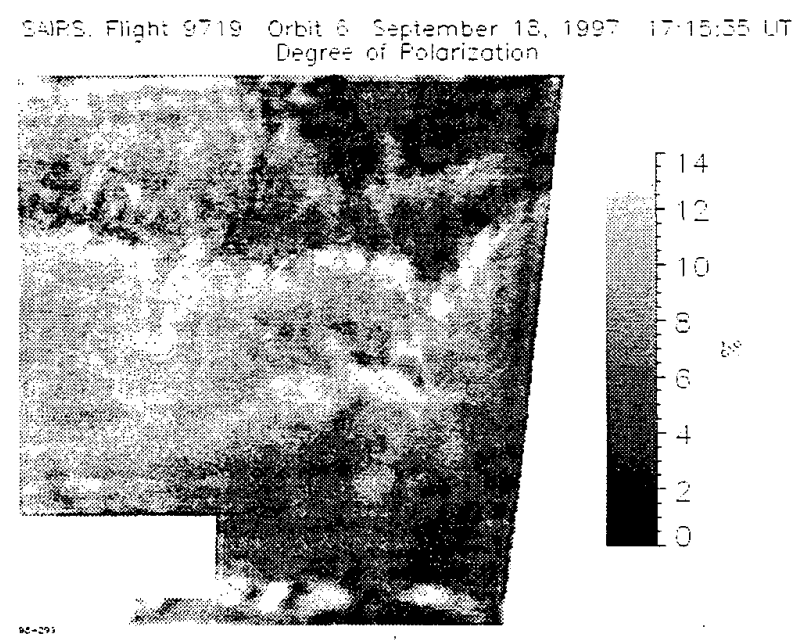

Figure 16 Sample SAIRS degree of polarization image

The typical degree of polarization in this image is about $12 \%$ which is similar to the $10 \%$ polarization observed by an earlier investigation at $2.2 \mu \mathrm{m}$ [1]. While the degree of polarization is fairly constant in the scene, there are regions especially in the more shadowed parts of the scene where the degree of polarization is quite low. This is interpreted to be the result of indirect lighting from clouds in the foreground. This effectively increases the amount of scattering in these shadowed parts (i.e. scattering from the foreground cloud which is then scattered from the parts of the cloud in the shadows) which would be expected to decrease the degree of polarization.

Another interesting pair of total intensity and polarization images obtained by SAIRS is shown in Figures 17 and 18 respectively. The images were taken at about 17:16:52 UT and have a mean scattering angle of $146^{\circ}$. This image shows a more diffuse portion of the cloud deck where the foreground cloud is less than 5\% polarized. A neighboring cloud in the upper right of this image shows a noticeably greater degree of polarization of about $10 \%$. This seems to be the result of changes in the scattering properties of the clouds over a very small spatial scale independent of the scattering angle. These changes could be the result of differences in the scatterers' size distribution and/or phase.

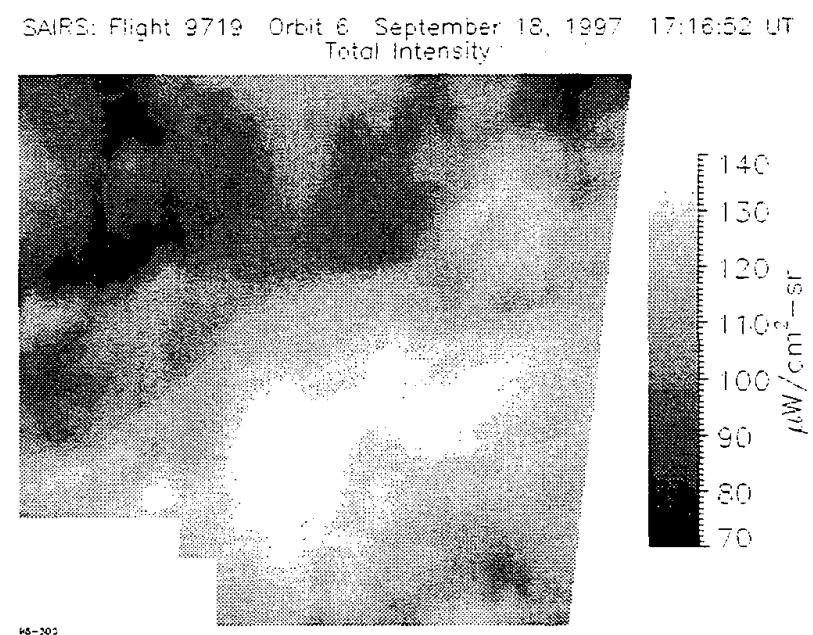

Figure 17 SAIRS total intensity image of cirrus-like clouds

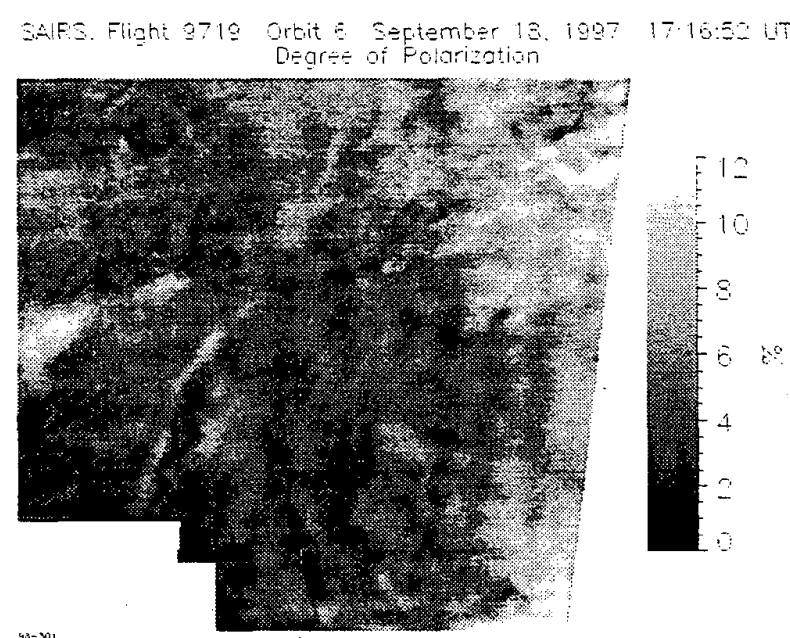

Figure 18 SAIRS degree of polarization image of cirruslike clouds

Because of the labor intensive nature of the procedure needed to register the images from a SAIRS polarization triplet, it was not possible to process all the SAIRS data into polarization images with the available time and resources. 
As a result only a small handful of image sets that displayed a large amount of polarization were processed in this way.

In order to obtain a more comprehensive look at how the scene's polarization changed in the SWIR as a function of scattering angle, a different processing technique was applied to the bulk of the SAIRS data. Instead of attempting to perfectly register all the features in a triplet of polarized filtered images, they were just roughly registered by simple translation. The regions of the three images that overlapped were identified and the data values of these image pixels were integrated to yield a triplet of single polarized filtered intensity measurements. The mean polarization of the scene was then determined by using just these measurements. In effect we used the SAIRS imager as a wide field photopolarimeter.

A comparison between the average degree of polarization derived from the fully registered SAIRS images and that calculated from this integrated image method were essentially identical. While the small-scale polarization information in the scene is lost by this processing method, it does allow us to make accurate scene-averaged polarization measurements with much less effort.
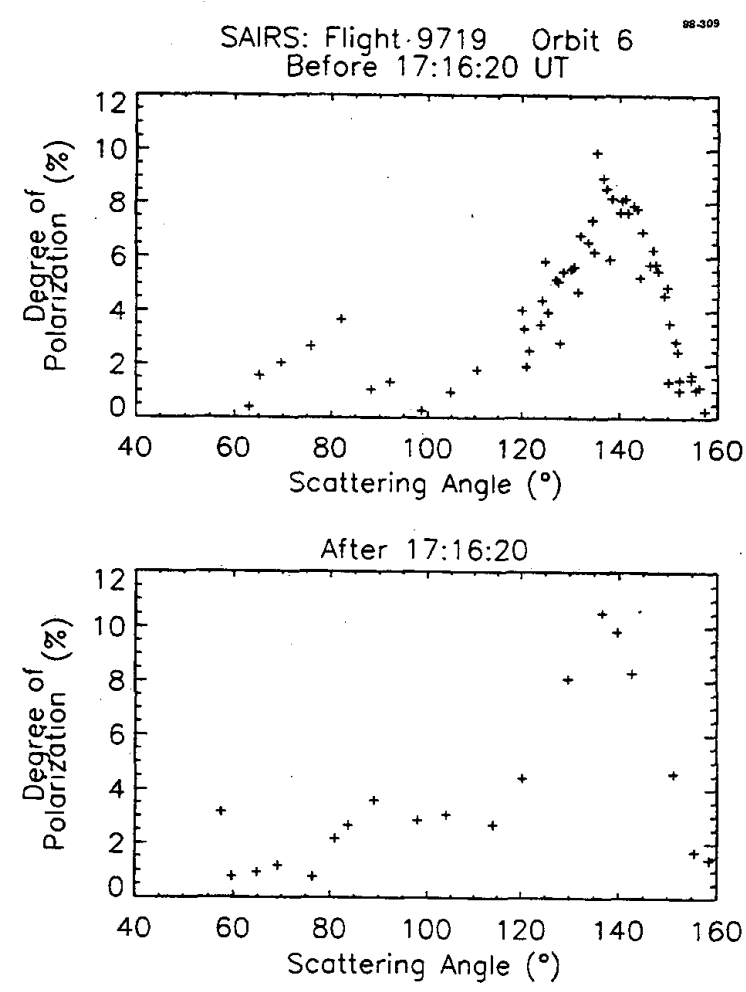

Figure 19 SWIR polarization curves for Flight 9719, Orbit 6 derived from SAIRS data

Plots of the average percent polarization for Orbit 6 of Flight 9719 is shown in Figure 19 as a function of scattering angle. Because data were obtained over nearly a complete circle of azimuth angles, scattering angles between about $60^{\circ}$ and $160^{\circ}$ were sampled twice during the orbit. In Figure 19, the top panel shows the polarization measurements made the first time the full range of scattering angles were sampled (before roughly 17:16:20 UT) while the bottom panel shows the second time the range was sampled. In this somewhat arbitrarily divided data set, the scene sampled in the top panel is dominated by clouds with a more cumulus-like appearance while those in the second set contain views of clouds with a more cirrus-like appearance.

In both sets of data there is a broad peak in polarization ranging from about $110^{\circ}$ to $160^{\circ}$ with the peak occurring near $137^{\circ}$. This angle seems to correspond to the primary rainbow at this wavelength. At scattering angles in the $60^{\circ}$ to $105^{\circ}$ range, the details of the polarization versus scattering angle function are distinctly different in the two data sets. These differences are considered to be real and probably reflect localized changes in the composition or scattering properties of the clouds.

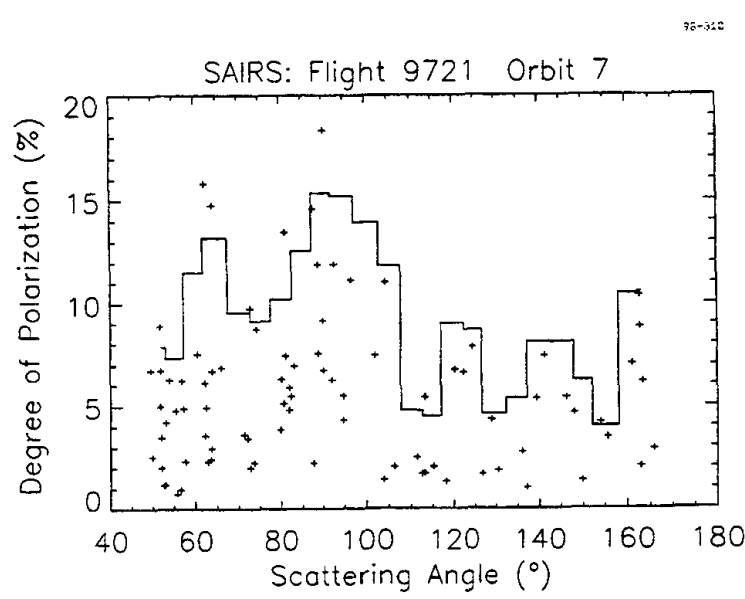

Figure 20 SWIR polarization data from Flight 9721, Orbit 7 with $90 \%$-confidence upper limit shown

Polarization measurements made by SAIRS during Orbit 7 of Flight 9721 shown in Figure 20 have a totally different character and seem to be dominated by noise. A detailed statistical analysis of the distribution of data points allowed us to derive a $90 \%$-confidence level upper limit for the degree of polarization assuming that the data points have a Gaussian distribution (modified by the fact that negative polarization values are not possible with our current algorithm).

The analysis indicates that there is a $90 \%$ probability that the degree of polarization is less than $10 \%$ to $15 \%$ at scattering angles in the $50^{\circ}$ to $100^{\circ}$ range and less than $5 \%$ to $10 \%$ in the $100^{\circ}$ to $165^{\circ}$ range. If the cloud deck observed during this orbit had the same characteristics as those observed during Orbit 6 of Flight 9719, the peak near $137^{\circ}$ should have been clearly visible. Obviously the differences in the scattering properties of these two cloud 
decks observed by PEELS have a profound effect on the observed degree of polarization.

\section{Visible Wavelength Results}

Analysis of the polarization imagery from MAVIS was confined primarily to data collected during Orbit 6 of Fight 9719. Because a triplet of polarization images was acquired at video rates, MAVIS images do not display the parallax effects that plagued the SAIRS images. As a result, a triplet of polarized-filtered MAVIS images could be quickly registered by simply translating the images and processing the portions of the calibrated images that overlapped. As a result, virtually all of the MAVIS imagery was amenable to detailed analysis using semi-automated techniques.

During Orbit 6 , the instrument was locked in position so that it was viewing approximately perpendicular to the aircraft's direction of travel and about $12^{\circ}$ above the aircraft reference frame's horizontal. As a result, the analyzed MAVIS imagery did not overlap with that of SAIRS (which was viewing about parallel with the aircraft's horizontal) or with regions sounded by PEELS (which was pointed $10^{\circ}$ below the horizontal). While with hindsight it would have been desirable to have coaligned these instruments, such coordination was not a requirement at this early stage of our investigation.

Nonetheless, the MAVIS observations were of great value in gaining additional insights into the scattering properties of the cloud deck as a whole and their spatial variability. One fortuitous result of the $+12^{\circ}$ elevation angle offset is that it allowed MAVIS to obtain comprehensive polarization and color imaging data at visible wavelengths through a range of elevation angles that included the apparent horizon (as defined by the top of the cloud deck) and the clear sky above the clouds even while the aircraft was in a banking turn throughout an orbit.
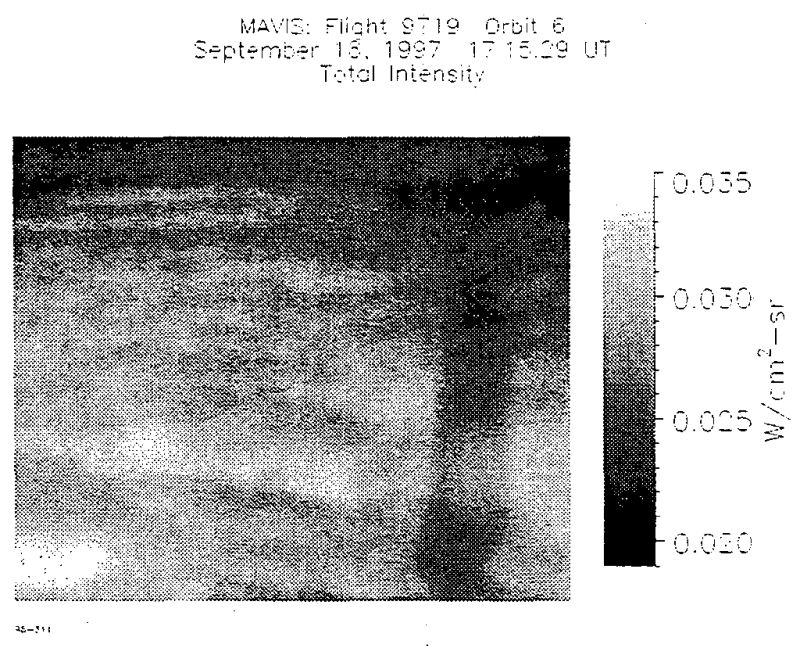

Figure 21 Typical MAVIS total intensity image from Flight 9719 , Orbit 6
A typical MAVIS image of the scene's total intensity (i.e. the sum of the polarized and unpolarized light) is shown in Figure 21. It shows the cumulus-like stratus cloud deck (as viewed at 17:15:29 UT) typical for the first half of Orbit 6 from an elevation angle of about $-9^{\circ}$ to almost $-1^{\circ}$. The image includes the horizon as well as a portion of the clear sky above the clouds which appears dark in this view. The scattering angle ranges from about $126^{\circ}$ in the upper left corner of the image to $137^{\circ}$ in the lower right corner.

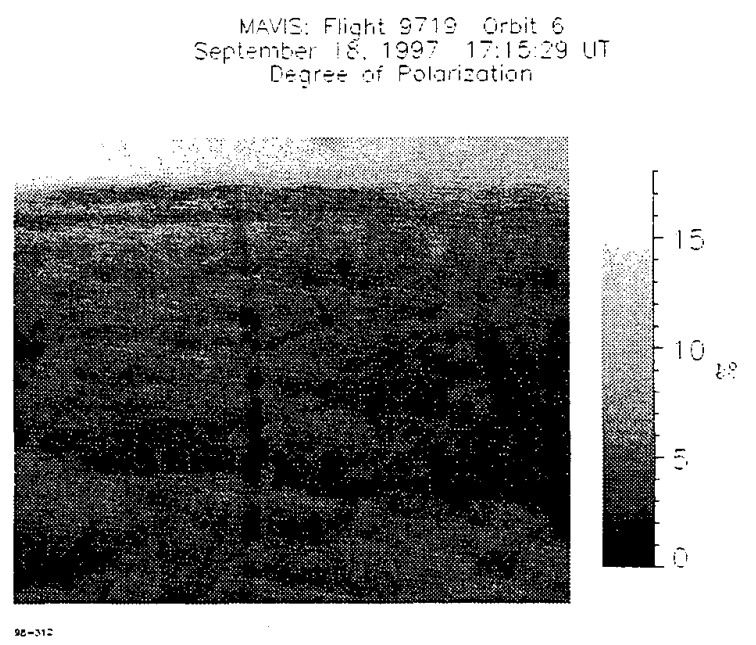

Figure 22 Typical MAVIS degree of polarization image from Flight 9719, Orbit 6

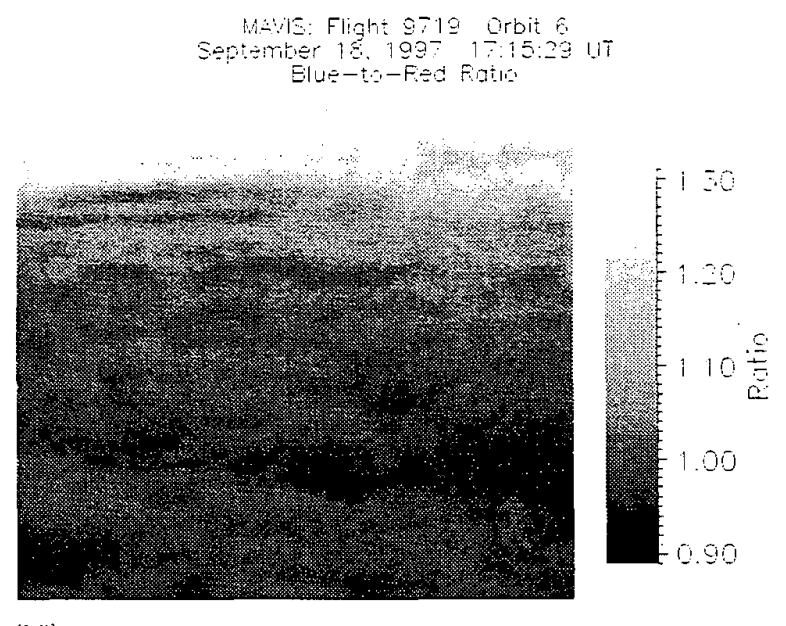

Figure 23 MAVIS blue-to-red ratio image

The corresponding degree-of-polarization image is shown in Figure 22. While the stratus clouds near the foreground typically display a few percent polarization, the light from the clear sky above the horizon is as much as $17 \%$ polarized as viewed here. This enhanced polarization is believed to be the result of scattering in the clear air above the cloud deck. A careful analysis of this image also shows that the degree of polarization systematically increases with elevation angle (as well as with the path length between the clouds and 
aircraft) hinting that Mie or Rayleigh scattering makes an important contribution to the scene's degree of polarization at visible wavelengths.

Further evidence for this hypothesis comes from an analysis of the scene's color. The ratio between blue- and redfiltered images obtained almost simultaneously with Figure 21 is shown in Figure 23. In this representation, the parts of the scene that are bluer appear brighter. It is immediately apparent that the clear sky above the clouds is the bluest and that the scene appears to become increasingly red with decreasing elevation angle. A two-dimensional histogram of the scene's percent polarization versus the blue-to-red ratio is shown in Figure 24. The strong positive correlation between the scene's blueness and degree of polarization is strong evidence for Mie or Rayleigh scattering.

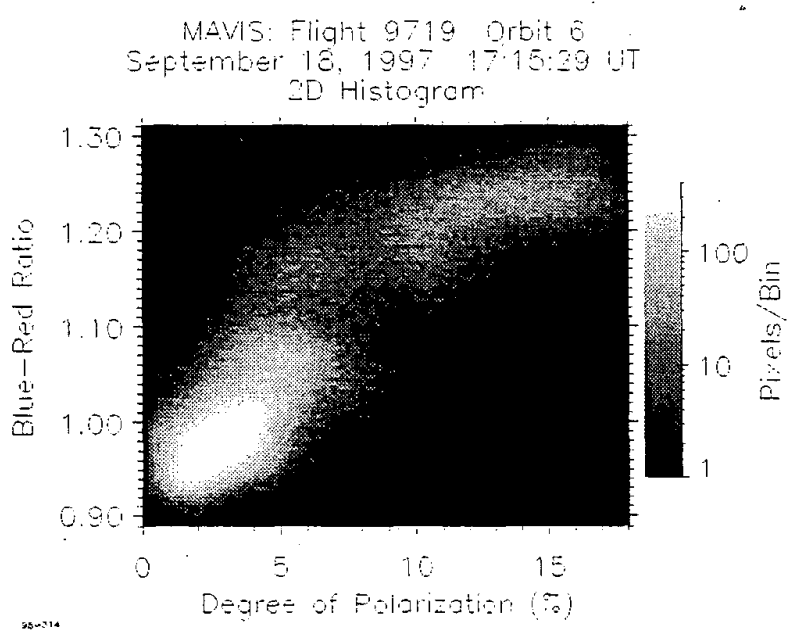

Figure 24 2D histogram of color ratio versus degree of polarization in MAVIS images

The effects of clear air scattering were not readily observed in the previously discussed SAIRS data for two reasons. First SAIRS consistently sampled lower elevation angles (where the clear air path length is shorter and scattering weaker) than MAVIS owing to the latter's pointing offset. By far the most important factor is that of wavelength. Because of the $1 / \lambda^{4}$ dependence on the strength of Rayleigh scattering, SAIRS would be expected to observe less than $0.2 \%$ of the Rayleigh scattering evident at visible wavelengths.

Because of the presence of scattering in the MAVIS images, the degree of polarization in the scene at visible wavelengths is now a function of not only the scattering angle but the elevation angle as well. In order to gain additional insights into the scene's polarization properties throughout the range of angles sampled, the MAVIS images were remapped into scattering angle-elevation angle space. Images obtained during the first and last half of the orbit (i.e. before and after about 17:16:20 UT respectively) were averaged to yield two "maps" of the cloud deck's averaged degree of polarization as a function of scattering angle and elevation angle. Plots of the resulting polarization curves for selected elevation angles are shown in Figures 25 to 27.

The elevation angle dependence on the degree of polarization resulting from scattering in the clear atmosphere above the clouds are immediately apparent. The polarization curve for an elevation angle of $-1^{\circ}$ in Figure 25 , which has the greatest effective slant range and should be free of any contributions from the cloud. It displays a broad peak near a scattering angles $105^{\circ}$.

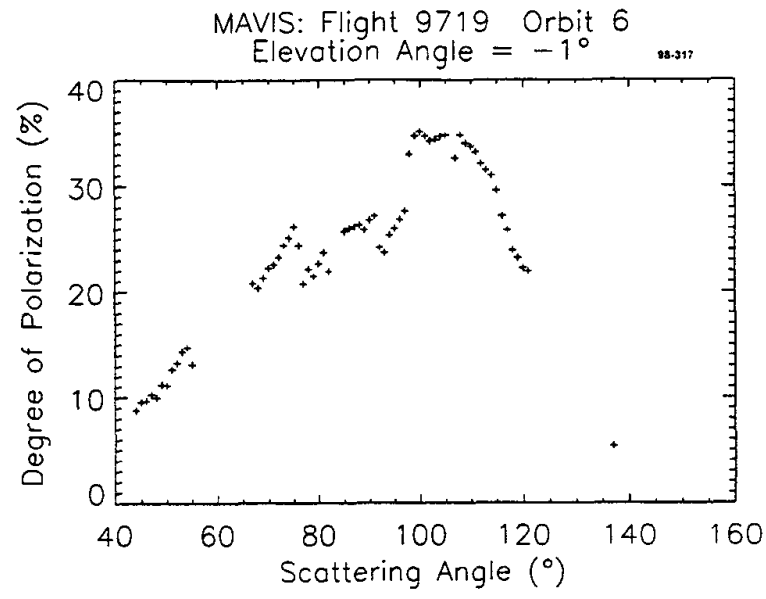

Figure 25 Degree of polarization versus scattering angle above cloud deck from MAVIS data

The pair of polarization curves for the lower elevation angles during the first half of Orbit 6 in Figure 26 (which was dominated by cumulus-like stratus clouds) both show the same basic shape that becomes somewhat flattened with decreasing elevation angle. These curves are probably the result of the scattering properties of the clouds in combination with an elevation angle-dependent contribution from clear air scattering.

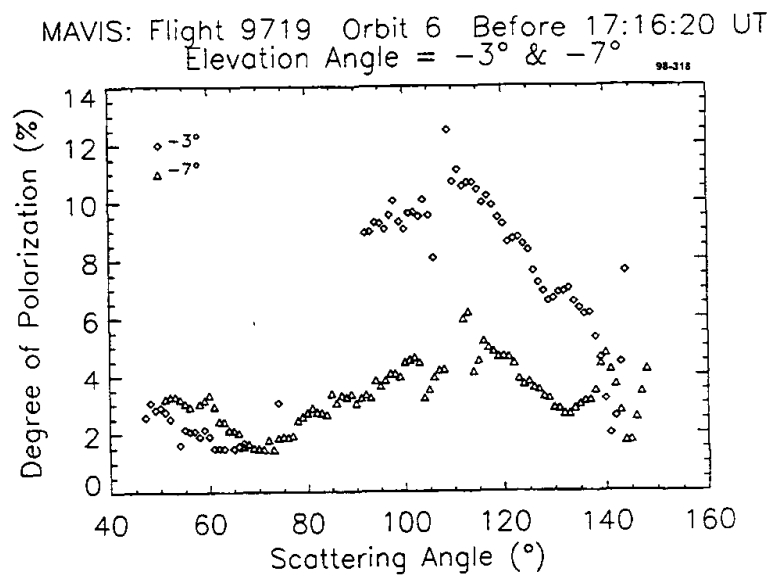

Figure 26 Degree of polarization versus scattering angle at elevation angle of $-3^{\circ}$ and $-7^{\circ}$ during first half of Orbit 6 on Flight 9719 
The curves for the second half of the orbit in Figure 27 (which is dominated by stratus clouds with a more cirruslike appearance) have a very different character from those of the first half. It is unclear whether the structure observed here is real or if it is the result of localized regions in the cloud deck with anomalous scattering properties. Once again we must be observing the elevation angle dependent effects of scattering superimposed on the polarization curve resulting from scattering from the cirrus-like clouds themselves.

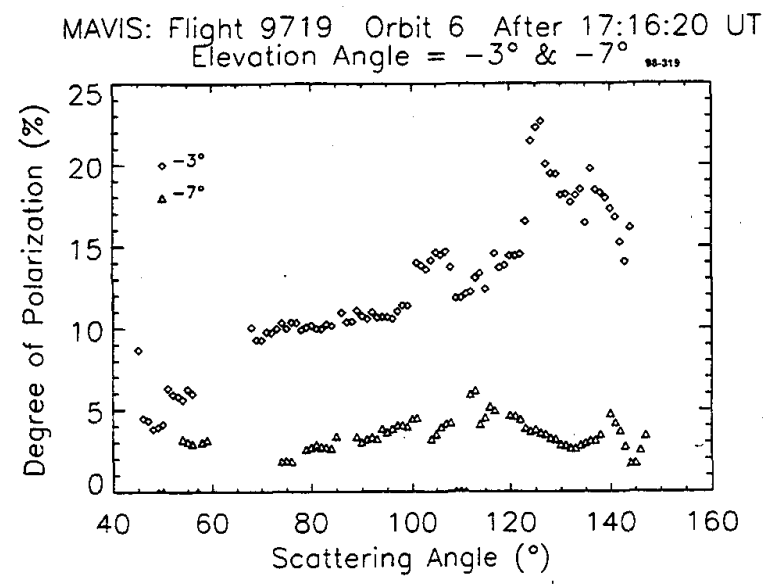

Figure 27 Degree of polarization versus scattering angle at elevation angle of $-3^{\circ}$ and $-7^{\circ}$ during second half of Orbit 6 of Flight 9719

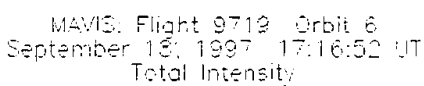

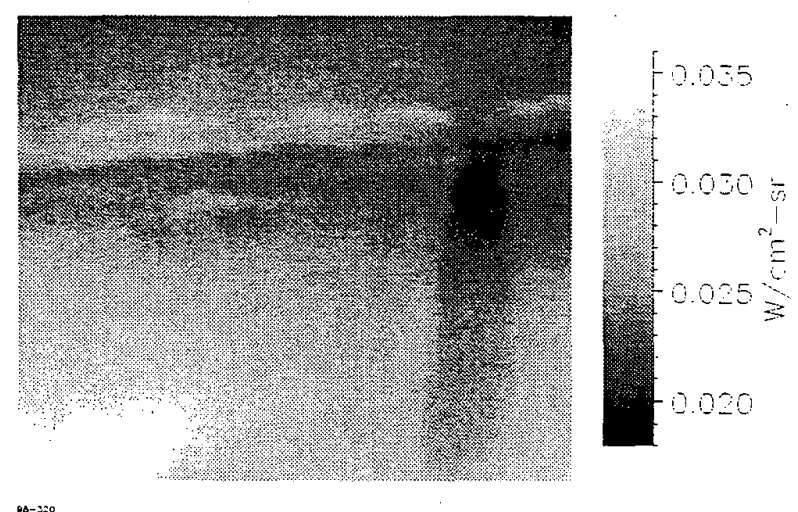

Figure 28 MAVIS total intensity image of featureless, cirrus-like clouds

The scene observed during the second half of the orbit was also notable in another way. The apparently featureless cloud deck shows distinct variations in its degree of polarization independent of the general dependence on elevation and scattering angles. This is clearly displayed in an example taken at 17:16:52 UT shown in Figures 28 and 29. In these images, the scattering angle varies from about $136^{\circ}$ in the upper right corner to almost $146^{\circ}$ in the lower left corner.
The total intensity image in Figure 28 shows a featureless deck of clouds stretching out to the horizon where more cumulus-like clouds are apparent and above where the clear sky can be seen. In the corresponding image in Figure 29 showing the degree of polarization, we see that there is much more structure apparent.

Superimposed on the foreground clouds which are about $10 \%$ polarized, there are readily visible regions with cloudlike appearance where the polarization can exceed $20 \%$. SAIRS observed similar such juxtaposition of low and high polarization clouds like in Figure 18 which was acquired at about the same time as this MAVIS image. It is not known if the same phenomenon is responsible for what is being observed at these different wavelengths. At higher elevation angles a fairly sharp but irregularly shaped boundary is reached between the predominantly low- and highpolarization regions of the cloud deck.
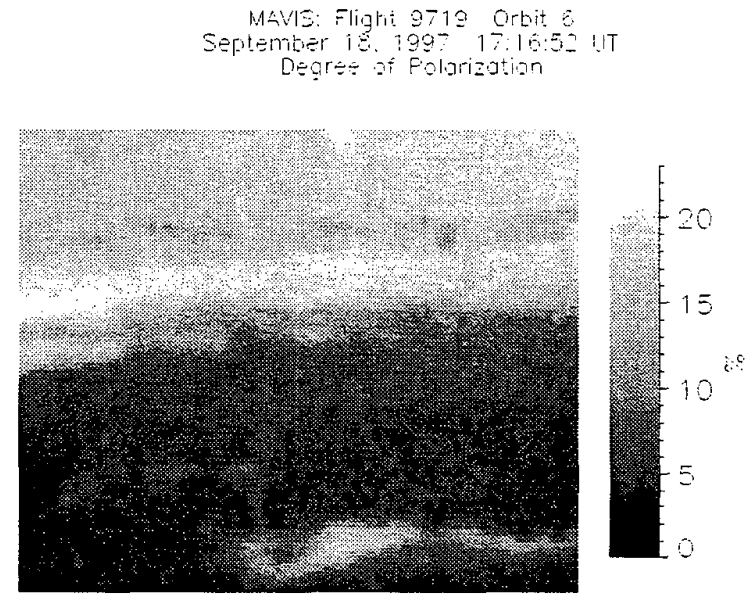

$95-\div:=$

Figure 29 MAVIS degree of polarization image showing structure in apparently featureless, cirrus-like clouds

The transitions seen in the cirrus-like cloud deck are too sudden to be caused by scattering in a clear, presumably homogeneous sky above the clouds. There is also no hint of any sharp transitions in the total intensity image of the scene. These highly polarized parts of the scene must correspond to regions with drastically different scattering properties that affect only the degree of polarization but not the total amount of reflected light. These distinctly different types of clouds are all lumped together in the polarization curves in Figure 27 and could be responsible for some of the structure observed in those curves. Still, these images do show that the scattering properties of even an apparently homogeneous cloud deck can be variable on a range of spatial scales.

One other set of MAVIS data that was analyze was images of a solar glint obtained during Orbit 3 of Flight 9721 at about 13:54:28 UT. These glints were actively tracked by the instrument operators when visible in an effort to gather 
data. The total intensity image derived from MAVIS data in Figure 30 shows the glint in a bank of cirrus-like stratus clouds when the Sun was at an elevation angle of about $11^{\circ}$. As discussed earlier, measurements made by PEELS showed this cloud deck to be fairly optically thin but quite deep. The image of the glint seems to be highlighting some underlying cumulus-like structure within the cloud deck which is attenuating the glint. The hints of a cloud layer about $1.3 \mathrm{~km}$ below the visible cloud tops in the lidar soundings during this flight may be from similar structures elsewhere in the cloud bank.
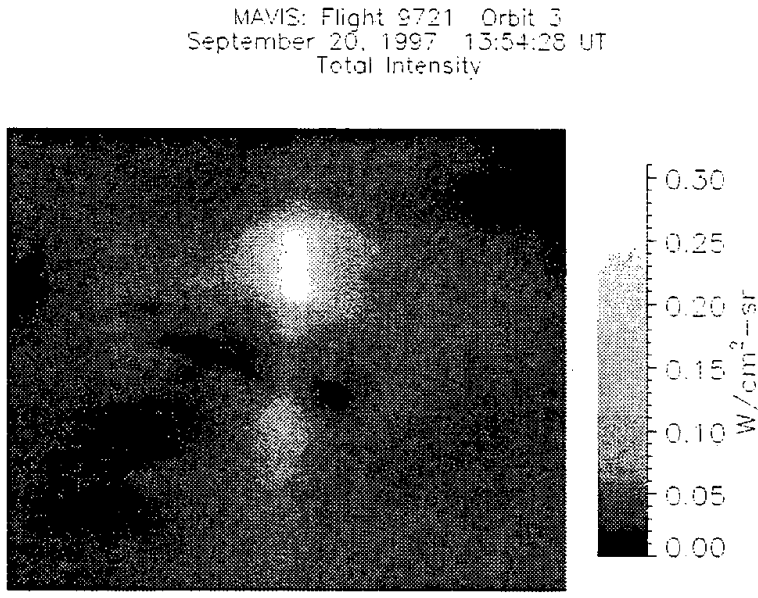

is -215

Figure 30 MAVIS total intensity image of Sun glint
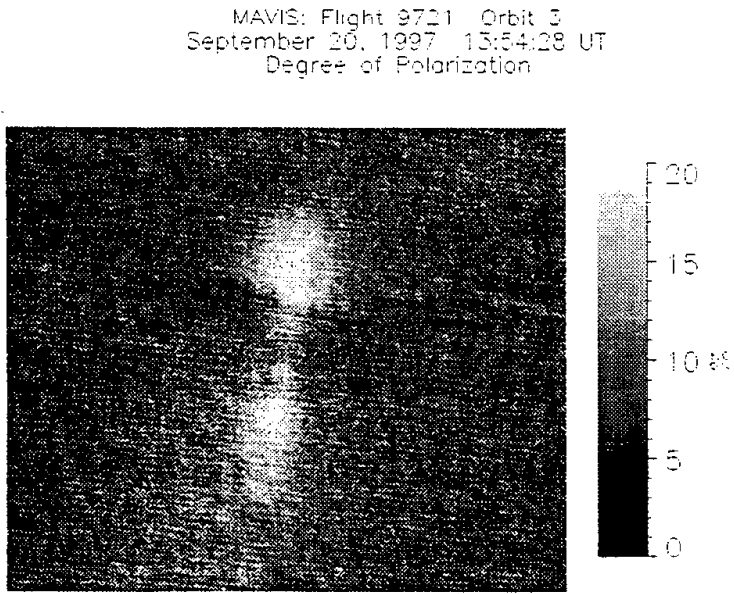

Figure 31 MAVIS degree of polarization image of Sun glint

The corresponding degree of polarization image of the Sun glint is shown in Figure 31. With a scattering angle ranging from $20^{\circ}$ at the top of the image to almost $29^{\circ}$ at the bottom, the background clouds exhibits less than $5 \%$ polarization and is lost in the image noise. The glint however is highly polarized in comparison and displays as much as $20 \%$ polarization. This image also shows signs of the cloud structure seen in the total intensity image of the glint.
SAIRS also obtained images of this glint but no usable polarization measurements could be derived from them because the cloud structure superimposed on the glint combined with aircraft motion caused the scene to change more quickly than data could be taken. Attempts to acquire data of the bright glint using the interferometers also proved to be unsuccessful for similar reasons.

\section{COMPARISON OF THEORY \& OBSERVATIONS}

With this initial set of measurements, it is now possible to compare our theoretical predictions with actual observations. While the computer codes used for our models are constantly evolving, the SWIR measurements made by SAIRS offer some interesting insights.

Figure 32 shows a plot of the absolute value of the degree of polarization predicted by our computer models for cloud composed of water and ice in the wavelength band observed by SAIRS (i.e. between $2.33 \mu \mathrm{m}$ and $2.65 \mu \mathrm{m}$ ). In both simulations, a population of spherical particles with a continuous, Gaussian-like size distribution with a mean diameter of $10 \mu \mathrm{m}$ was assumed. In Figure 32 only the absolute value of the degree of polarization is plotted to facilitate a comparison with our observations (whose calculation is currently limited to yielding only positive values).

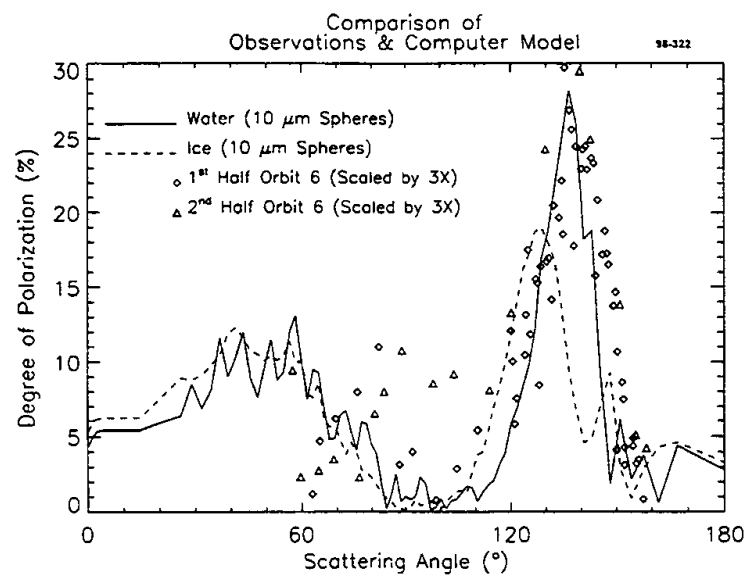

Figure 32 Comparison of SWIR observations and computer model predictions for the absolute value of $P$

Also plotted in Figure 32 are the data obtained by SAIRS during both halves of Orbit 6 of Flight 9719 . One thing that was immediately apparent when comparing the model's curves with the actual data was the fact the polarization signature observed by SAIRS was about a factor of 3 smaller. The actual data values have been increased by a factor of three in this plot to facilitate comparisons between the predictions and actual data.

The source of this magnitude difference is probably the result of several factors. First, the SAIRS data was 
corrected for the filter's spectral response function during calibration while the computer model assumed an idealized, top-hat shaped response curve. Even slight errors in either approach can yield large differences in the results especially in this region of the spectrum (i.e. on the shoulder of the 3 $\mu \mathrm{m}$ water absorption band).

The model also made the assumption that the cloud deck being observed is horizontal with no vertical relief. As can be seen in many of the SAIRS and MAVIS images, this is not always the case. Shadowed parts of the cloud scene have a relatively low degree of polarization as was seen in Figure 16. The inclusion of these regions in our analysis certainly decreases the average degree of polarization in the scene. Looking only at the directly illuminated portions of the scene in Figure 16, we find that the typical $12 \%$ polarization value is close to the $15 \%$ predicted by theory for this scene's scattering angle of $143^{\circ}$.

The lack of any detectable polarization signature during Orbit 7 of Flight 9721 hints that a simple model for water or ice clouds with a mean spherical particle size of $10 \mu \mathrm{m}$ is not a good fit for this cloud deck. Models for ice clouds with mean particle sizes of $20 \mu \mathrm{m}$ to $40 \mu \mathrm{m}$ can not be excluded however.

While the magnitude of the observed polarization signature differs greatly from that predicted by our models, the basic shape of the curves are gratifyingly similar especially at higher scattering angles. In particular, the curve for a water cloud is a fairly good match to the data from Orbit 6 of Flight 9719. Unfortunately lidar and spectral data indicate that ice clouds and clouds composed of a water-ice mixture were observed at this time.

Exactly how a mix of phase states or variations in the size distribution will affect the shape of the theoretical curves has yet to be investigated in detail. It is possible that a cloud composed of a water-ice mixture with more realistic (i.e. non-spherical) ice particles and a size distribution skewed more towards smaller particles would not only provide a better fit, but be in better agreement with lidar and spectral data. All in all, considering the substantial uncertainties that existed at the beginning of this endeavor, the computer models we have produced make predictions that bear a considerable resemblance to our observations.

Our computer model can also make predictions of polarization at visible wavelengths. But a comparison between these predictions at visible wavelengths and the data obtained by MAVIS is not as straightforward as it is with the SWIR SAIRS data. The presence of scattering in the clear atmosphere between the cloud deck and the aircraft adds a pronounced elevation angle-dependent polarization signature to the MAVIS data that is not included in our current computer models. As a result, a comparison between theoretical calculations and the MAVIS observations are dubious at best at this point. Further improvements in our models to take into account the effects observed by MAVIS will be incorporated in the future.

The MAVIS observations do show, however, that the scattering properties of the cloud deck are highly dynamic and spatially variable. Better coordination of the observations during the 1998 FISTA flights that will allow simultaneous observations by multiple instruments operating over a wide range of wavelengths promise to yield a better characterization of the scattering environment as well as its temporal and spatial variability.

\section{CONCLUSIONS}

The data gathered during the 1997 FISTA flights has yielded a large body of usable polarization data near the 3 $\mu \mathrm{m}$ water absorption band. This data has not only proved to be useful in planning another set of FISTA flights in 1998, but has provided sorely needed data to verify the computer models we are developing. While a comparison between of the predictions made by our initial models and our observations have shown a number of differences, the similarities evident even at this early stage are encouraging. While more study is needed, it seems likely that using polarization measurements near infrared water absorption bands to differentiate between clouds of various types or scattering properties is possible

Of particular importance is the large body of data we have collected showing the potential variability of the scattering environment in even an apparently featureless deck of clouds. While additional analysis and new data will help in quantifying this variability, the observations to date show that it can be significant and its effects will need to be included in future, more advanced models.

\section{ACKNOWLEDGEMENTS}

The RAMOS research described herein is supported by BMDO under Contract No. HQ 0006-97-D-0002 to the Space Dynamics Laboratory, Utah State University (SDL/USU). Visidyne, Inc. supported SDL/USU under subcontract \# C913686. The authors would like to thank the members of the FISTA flight team as well as Al Hurd, Dennis Villanucci, and Ted Zehnpfennig.

\section{REFERENCES}

[1] Kuo-Nan Liou, An Introduction to Atmospheric Radiation, International Geophysics Series Volume 26, Academic Press, 1980

[2] George W. Kattawar and Gilbert N. Plass, "Degree and Direction of Polarization of Multiple Scattered Light. 1: Homogeneous Cloud Layers", Applied Optics 11, 28512865, December 1972.

[3] James E. Hansen, "Multiple Scattering of Polarized Light in Planetary Atmospheres. Patr 2: Sunlight Reflected 
by Terrestrial Water Clouds", Journal of Atmospheric Science 28, 1400-1426, November 1971.

[4] R.A.R. Tricker, Introduction to Meteorological Optics, New York: Elsevier, 1970.

[5] Robert Greenler, Rainbows, Halos, and Glories, Cambridge, England: Cambridge University Press, 1980.

[6] Yoshihide Takano and Kuo-Nan Liou, "Solar Radiative Transfer in Cirrus Clouds. I - Single-Scattering and Optical Properties of Hexagonal Ice Crystals", Journal of Atmospheric Sciences 46, 3-19, January 1, 1989.

[7] Yoshihide Takano and Kuo-Nan Liou, "Solar Radiative Transfer in Cirrus Clouds. II - Theory and Computations of Multiple Scattering in an Anisotropic Medium", Journal of Atmospheric Sciences 46, 20-36, January 1, 1989.

[8] George M. Hale and Marvin R. Querry, "Optical Constants of Water in the 200-nm to $200-\mu \mathrm{m}$ Wavelength Range", Applied Optics 12, 555-563, March 1973.

[9] Joel W. Schaaf and Dudley Williams, "Optical Constants of Ice in the Infrared", Journal of the Optical Society of America 63, 726-732, June 1973.

[10] G.N. Plass and G.W. Kattawar, "Polarization of the Radiation Reflected and Transmitted by the Earth's Atmosphere", Applied Optics 9, 1122-1136, May 1970.

[11] George W. Kattawar and Gilbert N. Plass, "Degree and Direction of Polarization of Multiple Scattered Light. 2: Earth's Atmosphere with Aerosols", Applied Optics 11, 2866-2879, December 1972.

[12] James Thompson (Visidyne, Inc./Santa Barbara), Private communication, 1998

[13] K. Sassen, "The Polarization LIDAR Technique for Cloud Research: A Review and Current Assessment", Bulletin of the American Meteorological Society 72, 1848, 1991

\section{BIOGRAPHY}

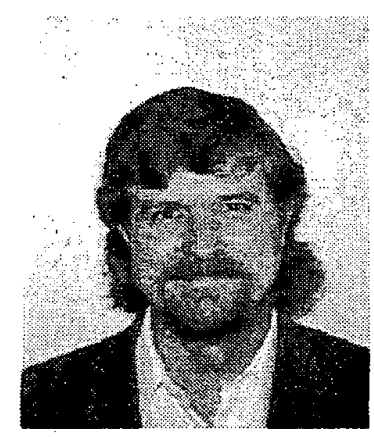

The lead author, Andrew J. LePage, is a Scientist at Visidyne, Inc. specializing in computer image processing and analysis of remote sensing data. Since his return to Visidyne in 1992, he has been involved in the development and implementation of image and optical data processing, analysis, and data visualization algorithms in support of a number of programs including RAMOS, EXPRESS, MSX,
CIRRIS, IBSS, and EXCEDE III. While with Visidyne from 1985 to 1987, he was active in the design and fabrication of $X$ ray and IR optical systems for high altitude and space applications as well as development of image processing techniques to support research in variable MTF optical systems. Between 1987 and 1992 Mr. LePage was a Senior Engineer at Digital Equipment Corp. where he did innovative work on non-contact electrical testing using optical and highpowered laser technologies. Mr. LePage received his BS in Physics from the University of Lowell in 1985. 